\title{
A Probabilistic Wavelet-Support Vector Regression Model for Streamflow Forecasting with Rainfall and Climate Information Input*
}

\author{
ZHIYONG LIU \\ Institute of Geography, Heidelberg University, Heidelberg, Germany \\ PING $\mathrm{ZHOU}^{+}$ \\ Department of Forest Ecology, Guangdong Academy of Forestry, Guangzhou, China \\ YINQIN ZHANG \\ College of Water Resources and Architectural Engineering, Northwest A\&F University, Yangling, China, and \\ Department of Agricultural and Biological Engineering, Purdue University, West Lafayette, Indiana
}

(Manuscript received 24 October 2014, in final form 26 March 2015)

\begin{abstract}
It is essential to explore reliable streamflow forecasting techniques for water resources management. In this study, a Bayesian wavelet-support vector regression model (BWS model) is developed for one- and multistep-ahead streamflow forecasting using local meteohydrological observations and climate indices including El Niño-Southern Oscillation (ENSO) and the Indian Ocean dipole (IOD) as potential predictors. To accomplish this, a two-step strategy is applied. In the first step, the discrete wavelet transform is coupled with a support vector regression model for streamflow prediction. The three key factors of mother wavelets, decomposition levels, and edge effects are considered in the wavelet decomposition phase when using the hybrid wavelet-support vector regression model (WS model). Different combinations of these factors form a variety of WS models with corresponding forecasts. The second step combines multiple candidate WS models with "good" performance via Bayesian model averaging. This integrates the predictive strengths of different candidate WS models, giving a realistic assessment of the predictive uncertainty. The new ensemble model is used to forecast daily and monthly streamflows at two sites in Dongjiang basin, southern China. The results show that the proposed BWS model consistently generates more reliable predictions for daily (lead times of 1-7 days) and monthly (lead times of 1-3 months) forecasts as compared with the best single-member WS models and the adaptive neuro-fuzzy inference system (ANFIS). Furthermore, the proposed BWS model provides detailed information about the predictive uncertainty.
\end{abstract}

\section{Introduction}

Effective and skillful streamflow forecasting is particularly important in improving flood prevention measures

\footnotetext{
* Supplemental information related to this paper is available at the Journals Online website: http://dx.doi.org/10.1175/JHM-D-14-0210.s1.

${ }^{+}$Current affiliation: Guangdong Provincial Key Laboratory of Biocontrol for the Forest Disease and Pest, Guangdong Academy of Forestry, Guangzhou, China.
}

Corresponding author address: P. Zhou, Guangdong Provincial Key Laboratory of Biocontrol for the Forest Disease and Pest, Guangdong Academy of Forestry, Guangzhou 510520, China.

E-mail: zhoupinger@gmail.com and optimizing the operation and management of available water resources. In the past few decades, data-driven models, for example, multiple linear regression (MLR), autoregressive moving average (ARMA), artificial neural network (ANN) models, and adaptive neuro-fuzzy inference system (ANFIS), have become popular for shortand long-term streamflow prediction (McKerchar and Delleur 1974; Kim and Barros 2001; Kisi and Cimen 2011; Nourani et al. 2014). These techniques have the advantages of minimum information requirements, ease of real-time implementation, and rapid development times (Moradkhani et al. 2004). Although data-driven models may not be able to provide physical interpretations and insights into catchment processes, they usually produce relatively skillful and accurate forecasts (Tiwari and 
Chatterjee 2010). Thus, they might be a suitable alternative to physical models, particularly for data-sparse basins.

Recently, the support vector regression (SVR) model, originating from support vector machines (SVMs), has been successfully applied for hydrological prediction (Liong and Sivapragasam 2002; Bray and Han 2004; Yu et al. 2006; Wang et al. 2009). Kisi and Cimen (2011) further developed SVR by coupling the wavelet transform technique for monthly streamflow forecasting, and they concluded that the hybrid wavelet-SVR (WS) model generated more accurate forecasts. The wavelet transform is a signal analysis technique that provides a time-frequency representation of a signal at different periods in the time domain (Daubechies 1990; Tiwari and Chatterjee 2010). In practice, it has been shown to be an ideal tool for analyzing nonstationary signals (e.g., the streamflow) that contain sharp changes and discontinuities (Liu et al. 2011; Quiroz et al. 2011). In the wavelet-based SVR model, the wavelet transform decomposes the original input data into several periodic components (subseries) of different resolutions, and these are then used as the new inputs of the SVR model. The wavelet-decomposed data can thus improve the ability of the forecasting model by capturing useful information at various resolutions (Tiwari and Chatterjee 2010). In addition to the conjunction with an SVR model, the wavelet transform has also been widely combined with other data-driven models such as ANN for hydrological simulations and predictions (Kim and Barros 2001). These studies all stated that the waveletbased forecasting model obtained superior results compared with traditional data-driven models.

However, some important issues concerning coupling SVR models with wavelet transforms need to be clarified and explored in detail. These issues involve the choice of a wavelet family, decomposition levels, and the influence of edges in the wavelet decomposition phase of the WS model. Although such aspects should necessarily be investigated when using the wavelet-based SVR model (or other wavelet-based models, e.g., waveletANN), they are often overlooked or determined based on personal preferences. Our earlier study (Liu et al. 2014) indicated that different combinations of these three issues may result in relatively significant differences in the performance of streamflow prediction, and that different hybrid models (wavelet-based SVR models) did not always outperform the original SVR model. It is thus crucial to consider and compare these three key factors when using wavelet-based data-driven models to obtain the "best" combination. However, choosing the single "best" WS model may exclude other competing combined models that exhibit similar overall performance but yield different forecasts for individual events, and may also not account for model uncertainty (Pokhrel et al. 2013). It is therefore desirable to build a general scheme with respect to the choice of wavelets, decomposition levels, and edges when using waveletbased forecasting models for streamflow prediction. This study seeks to develop a feasible alternative to establish an ensemble prediction using a candidate pool of different WS models.

The general multimodel ensemble technique combines multiple model forecasts using a set of deterministic weights, such as the simple arithmetic mean, Granger-Ramanathan mean, linear regression, and ANN-based weight estimating method (Granger and Ramanathan 1984; Ajami et al. 2007). However, weights determined by these approaches may take on arbitrary values that are unrelated to model performance. Furthermore, these multimodel techniques cannot produce ensemble predictions with uncertainty estimates (Duan et al. 1992). Bayesian model averaging (BMA) has been recently reported to be a more effective way of combining forecasts from multiple models (Raftery et al. 2005; Ajami et al. 2007; Liu and Gupta 2007; Luo and Wood 2008; Hsu et al. 2009; Pokhrel et al. 2013). BMA is a probabilistic multimodel averaging method that combines weighted candidate models and generates a realistic assessment of the predictive uncertainty (Hoeting et al. 1999; Duan et al. 2007; Franz et al. 2010; Wang and Robertson 2011). The BMA weights (likelihood measures) for each candidate model, being nonnegative and summing to 1 , represent each model's relative forecast skill, as they are based on the success frequency of the predictions that an individual model has made within the observations (Parrish et al. 2012). Over the last decade, the BMA technique has been applied in the field of hydrology, for combining hydrological groundwater models and generating an ensemble prediction from multiple conceptual rainfall-runoff models (Neuman and Wierenga 2003; Duan et al. 2007; Vrugt and Robinson 2007; Rojas et al. 2008; Liu et al. 2012). More recently, Rathinasamy et al. (2013) employed a single static Bayesian weight to combine each wavelet-based Volterra model for streamflow forecasting. These applications reflect the fact that the BMA-based multimodel forecasts outperform those produced from the traditional models.

The objective of this study is to develop a Bayesianbased WS model that can improve one- and multistepahead streamflow prediction and assess model uncertainty by using local meteohydrological observations and climate indices as potential predictors. To achieve this, we apply a two-step strategy that combines the strengths of the WS model and BMA to give a BWS model. First, we evaluate the forecasting skill of a variety of WS models by considering different combinations of mother 
wavelets, decomposition levels, and edge effects in the wavelet decomposition phase. Certain combinations illustrate the structural deficiencies in hybrid WS models. Second, we use BMA (allowing for dynamic model weights changing with respect to the hydrograph) to combine ensemble forecasts from the WS models with "good" performance and quantify the predictive uncertainty resulting from the structural discrepancies in individual models. The proposed approach is then applied to the forecasting of both daily and monthly streamflow. The forecasting skill of the proposed BWS model is compared with the best single-member WS model and ANFIS.

\section{Theoretical background}

\section{a. Support vector regression}

SVR was proposed by Drucker et al. (1997) and is used to describe regression with SVMs (Vapnik et al. 1997). The idea of SVR is based on the computation of a linear regression function in a high-dimensional feature space, where the input data are mapped via a nonlinear function (Yu et al. 2006; Wei and Roan 2012). Using mathematical notation, the regression function (SVR model) can be expressed as

$$
f(x)=[\mathbf{w} \cdot \Phi(x)]+b,
$$

where $x$ is the model input, $\mathbf{w}$ is the weight vector, $b$ is a bias, and $\Phi(x)$ indicates a mapping function that uses a nonlinear function to transform the nonlinear input to a linear mode in high-dimensional feature space (Kao et al. 2013). The quality of estimation is measured by the loss function. SVR uses a new type of $\varepsilon$-insensitivity loss function proposed by Vapnik et al. (1997):

$$
L_{\varepsilon}[f(x), y]= \begin{cases}|f(x)-y|-\varepsilon & \text { for }|f(x)-y| \geq \varepsilon, \\ 0 & \text { otherwise }\end{cases}
$$

where $y$ is the desired (target) output and $\varepsilon$ defines the region of $\varepsilon$ insensitivity and is the user-determined parameter (Wang et al. 2009). When the predicted value is within the band area, the loss is zero. On the contrary, if the predicted value falls outside the band area, the loss is equal to the difference between the predicted value and the margin (Kao et al. 2013).

The regression problem in Eq. (1) can be estimated by minimizing the following regularized risk function:

$$
R_{\mathrm{reg}}=C \frac{1}{n} \sum_{i=1}^{n} L_{\varepsilon}\left[f\left(\mathbf{x}_{i}\right), \mathbf{y}_{i}\right]+\frac{1}{2}\|\mathbf{w}\|^{2},
$$

where the first term is the empirical risk, the second term is the regularization term that determines the trade-off between the complexity and approximation accuracy of the regression model to ensure that the model possesses an improved generalized performance, and $C$ is a userdefined regularization constant that influences the trade-off between the regularization terms and empirical risk. The input vectors are $\mathbf{x}_{i}$ and the corresponding output values are $\mathbf{y}_{i}(i=1,2, \ldots, n)$.

Considering empirical risk and structure risk together, Eq. (3) can be transformed into the following constrained form using slack variables, which is used to allow some degree of slackness in constraint (Vapnik et al. 1997, 2000; Kao et al. 2013):

$$
\begin{gathered}
\operatorname{minimize} \frac{1}{2}\|\mathbf{w}\|^{2}+C \sum_{i=1}^{n}\left(\xi_{i}+\xi_{i}^{*}\right), \\
\text { subject to }\left\{\begin{array}{l}
\mathbf{y}_{i}-f\left(\mathbf{x}_{i}\right) \leq \varepsilon+\xi_{i} \\
f\left(\mathbf{x}_{i}\right)-\mathbf{y}_{i} \leq \varepsilon+\xi_{i}^{*} \\
\xi_{i} \geq 0, \quad \xi_{i}^{*} \geq 0, \quad i=1, \ldots, n
\end{array}\right.
\end{gathered}
$$

where $\xi_{i}$ and $\xi_{i}^{*}$ are the positive slack variables used to measure the deviation of training samples outside the $\varepsilon$-insensitivity zone.

Equation (4) is a constrained quadratic optimization problem (Kao et al. 2013). This can be solved by applying Lagrangian theory and the Karush-Kuhn-Tucker complementarity conditions (Azamathulla and $\mathrm{Wu} 2011$ ). Finally, the general form of the SVR-based regression function can be expressed as

$$
f(x)=\sum_{i=1}^{n}\left(\alpha_{i}-\alpha_{i}^{*}\right) K\left(x, \mathbf{x}_{i}\right)+b,
$$

subject to $0 \leq \alpha_{i} \leq C, \quad 0 \leq \alpha_{i}^{*} \leq C$,

where $\alpha_{i}$ and $\alpha_{i}^{*}$ are the Lagrangian multipliers and $K\left(x, \mathbf{x}_{i}\right)$ is the kernel function expressed by $K\left(x, \mathbf{x}_{i}\right)=\Phi(x) \Phi\left(\mathbf{x}_{i}\right)$. Kernel functions are used to change the dimensionality of the input space, resulting in a more confident regression (Azamathulla and Wu 2011). Several kernels are available in SVR, including linear, polynomial, sigmoid, and radial basis function (RBF) kernels (Yu et al. 2006). Dibike et al. (2001) compared different SVR kernels for rainfall-runoff modeling and concluded that the RBF is superior to other kernel functions. The output from many SVM-based hydrological forecasting applications suggests that RBFs result in favorable performance (Liong and Sivapragasam 2002; Yu et al. 2006; Kisi and Cimen 2011). Thus, in this study, we use the RBF kernel. It can be written as $K\left(x, \mathbf{x}_{i}\right)=\exp \left(-\left\|x-\mathbf{x}_{i}\right\|^{2} / 2 \sigma^{2}\right)$, where $\sigma$ represents the width of the RBF (Vapnik 2000). The SVR parameters were optimized using a two-step grid search method. Detailed information about this optimization method and the SVR model is given by Yu et al. (2006) and Hsu et al. 
(2010). We implement the SVR models based on the LIBSVM program developed by Chang and Lin (2001).

\section{b. Wavelet analysis}

The wavelet transform has become an effective tool for the analysis, denoising, and compression of signals and images. The objective of the wavelet transform is to obtain a complete time-scale representation of localized and transient phenomena occurring on different time scales (Labat et al. 2000). Signals are decomposed into a variety of components at different resolution levels using a wavelet function, which is also called the mother wavelet (Tiwari and Chatterjee 2010). When compared with the classical Fourier transform, which can only provide the frequency information of a signal, the main advantage of the wavelet transform is its ability to simultaneously gain information on the time, location, and frequency of a signal. In general, wavelet transforms can be performed using two approaches: the continuous wavelet transform (CWT) and discrete wavelet transform (DWT; Nalley et al. 2012). The construction of the CWT inverse is more complicated, requiring a significant amount of computation time (Partal and Küçük 2006). In contrast, the DWT needs less computation time and is simpler to develop than the CWT. The process of transformation in DWT is simplified and the amount of work is reduced; yet, it still generates a very efficient and accurate analysis (Nalley et al. 2012). This is attributed to the fact that the DWT is normally based on dyadic calculations (i.e., integer powers of two) of the position and scale of a signal (Chou 2007). DWT is especially useful when the signal contains jumps or shifts (Nalley et al. 2012). The DWT takes the following form (Partal and Küçük 2006):

$$
\psi_{(j, k)}\left(\frac{t-\gamma}{s}\right)=\frac{1}{s_{0}^{j / 2}} \psi\left(\frac{t-k \gamma_{0} s_{0}^{j}}{s_{0}^{j}}\right),
$$

where $\psi$ is the wavelet function, $t$ is time, $\gamma$ is the translation factor (time step) of the wavelet over the time series, $s$ indicates the scale (scale factor), $j$ is an integer that determines the dilation, $k$ is an integer that determines the translation, $s_{0}$ is a specified fixed dilation step greater than 1 , and $\gamma_{0}$ denotes the location parameter (must be greater than zero). The most common and simplest choice for the parameters is $s_{0}=2$ and $\gamma_{0}=1$ (Mallat 1989).

In DWT, a time-scale signal is obtained using digital filtering techniques. The original time series are passed through high- and low-pass filters and separated into different scales (Kisi and Cimen 2011). The time series are decomposed into two parts: the approximation that comprises low frequencies (large-scale components) and their trend, and details comprising high frequencies (small-scale components) and fast events.

\section{Case study area and data}

To test the forecasting ability of the proposed approach, we selected two sites for a case study: Longchuan (upper Dongjiang River) and Yuecheng (Xinfeng River, a tributary of the Dongjiang River) in the Dongjiang basin $\left(22^{\circ} 34^{\prime}-25^{\circ} 12^{\prime} \mathrm{N}, 113^{\circ} 24^{\prime}-115^{\circ} 53^{\prime} \mathrm{E}\right)$, southern China, which has a drainage area of $27040 \mathrm{~km}^{2}$. The basin is also an important subbasin of the Pearl River basin, which is the second-largest river in China in terms of annual runoff generation (Niu 2013). Dongjiang basin is located in a monsoon-dominated climate (Wu and Chen 2013). The annual mean temperature is approximately $20^{\circ}-22^{\circ} \mathrm{C}$, and the mean annual precipitation varies between 1500 and $2400 \mathrm{~mm}$ (Lin et al. 2011). The location of two gauging sites is shown in Fig. 1.

The local observed rainfall and streamflow records from these sites were used as the potential predictors for one- and multistep-ahead streamflow forecasting. The available data span the period from 1989 to 2011. The meteohydrological data were provided by Guangdong Hydrological Bureau (China). Von Neumann independence, the Mann-Whitney homogeneity tests, and Mann-Kendall trend analysis were applied to test the quality of the data (Bartels 1982; Alexandersson 1986). The results of these tests indicated that the meteohydrological data of the two sites in the present study are independent, homogeneous, and stationary (no trends and jumps found) at a given significance level ( $\alpha=5 \%)$. It should be mentioned here that a better prediction model could be established by providing more meteorological data (e.g., maximum temperature, mean temperature, air pressure, as well as rainfall data from more sites) as the potential predictors, but such data are not available for the current study. Additionally, we considered climate indices including El Niño-Southern Oscillation (ENSO) and the Indian Ocean dipole (IOD) from 1989 to 2011 as predictors for monthly streamflow forecasting, as some existing studies have indicated possible teleconnections between rainfall patterns (or streamflow) and climate indices in the Pearl River basin or the current study basin (Wang et al. 2010; Chen et al. 2011; Niu 2013). The multivariate ENSO index (MEI) was used to represent ENSO activity in this study, as this provides a more complete and flexible description of the ENSO phenomenon than singlevariable ENSO indices (e.g., Niño-3 SST and Niño-3.4 SST; Wolter and Timlin 2011). Monthly data were 


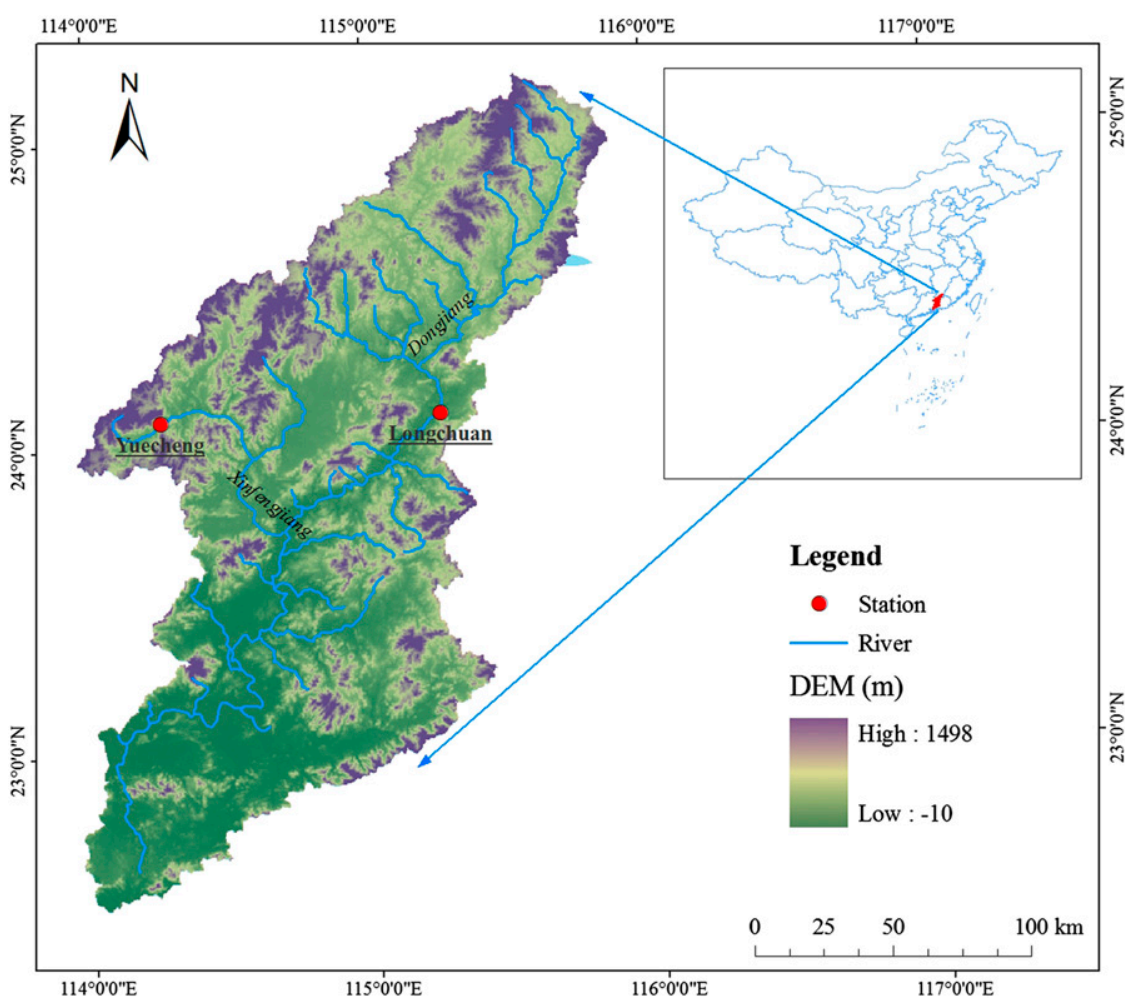

FIG. 1. Location of the two sites used for this study.

attained from NOAA (2015). The intensity of the IOD is represented by the dipole mode index (DMI; Saji et al. 1999; Saji and Yamagata 2003). The monthly DMI data were extracted from JAMSTEC (2012). The cross correlation between climate indices and monthly streamflows at two sites is given in this study (see Table S1 in the supplemental material). For lead times of 1-3 months, positive correlations are found at both sites.

In this study, data from the 19-yr period 1989-2007 were used for model calibration or development, and the remaining data (2008-11) were subsequently reserved for verifying or evaluating the forecast performance.

\section{Development of the multimodel approach}

\section{a. Candidate WS models}

We established a variety of WS models by coupling the DWT and SVR approaches, and we generated individual forecasts for the model ensemble and "best" model selection. A schematic of the WS model with the potential predictors and the ensemble BWS model given by a Bayesian technique is summarized in Fig. 2. In the WS model, the original time series of potential predictors are decomposed into a number of periodic components (subseries) at different resolution levels via DWT. Each component plays a different role in the original time series, and the behavior of each subseries is distinct (Wang and Ding 2003; Kisi and Cimen 2011). Since all subseries are equally important and contain specific information in the original data, averaging or optimizing the input of certain subseries is a potentially diminutive approach (Adamowski and Sun 2010). In the current study, all subseries at time $t-n$ (where $n$ indicates the lead time) were used as inputs to the SVR model. The output of the model is the original streamflow time series at time $t$. Accordingly, the new WS model takes advantage of both the wavelet and SVR techniques.

In the WS model, several key factors of the wavelet decomposition phase that may significantly affect the forecasting accuracy are considered and compared. This step is often missing in some existing wavelet-based streamflow forecasting literatures (e.g., Kisi and Cimen 2011). Such factors involve the mother wavelet, decomposition level, and edge effect in the DWT (Liu et al. 2014)

Choosing the mother wavelet is an important task in wavelet-related analyses (Sang et al. 2013). In fact, the forecasting skill in the WS model generally varies depending on the wavelet used. In this study, we consider a variety of wavelet families that are commonly used in hydrometeorological wavelet-based studies and attempt 


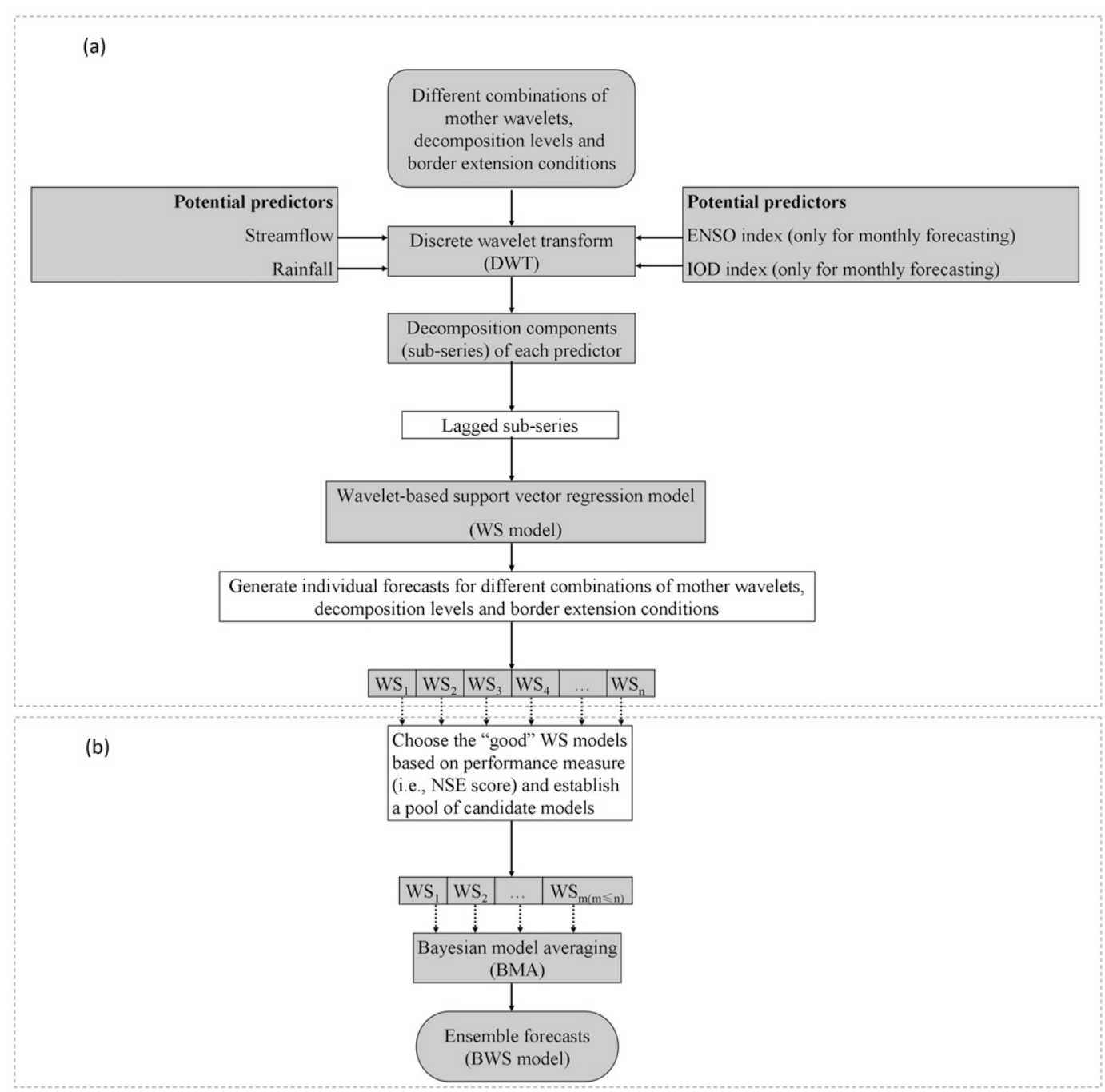

FIG. 2. Schematic of the (a) WS and (b) BWS models.

to reveal their performance discrepancies (Nourani et al. 2009b; Maheswaran and Khosa 2012a; Maheswaran and Khosa 2012b; Nalley et al. 2012). We examine the Daubechies (db4-10), Symlet (sym2-8), and Haar wavelets, giving a total of 15 wavelets.

To preserve information and reduce distortion in the original time series, the selection of an appropriate decomposition level is also important. The number of decomposition levels is generally based on the original time series length (Nalley et al. 2012). The method used to determine the number of decomposition levels is given by the formula $L=[\log (N)]$, where $L$ [the nearest integer to $\log (N)$ ] denotes the decomposition level and $N$ is the number of time series data (Nourani et al. 2009a; Tiwari and Chatterjee 2010). For the 23-yr period considered in our case study, there are $N=8400$ data points for the daily time series, so $L=3[\log (8400)=3.92]$. For monthly time series, the resulting maximum level of decomposition is $2[\log (276)=2.44]$.

Additionally, for time series of limited length, the convolution process cannot proceed at both ends of the series, because no information is available outside these boundaries when implementing the DWT (Su et al. 2011). Thus, the border effect should also be taken into consideration, and an extension at both borders is required. Commonly used border conditions include symmetrization (assuming that signals outside the original support can be recovered by symmetric boundary replication), zero padding (padding the signal with zeros beyond the original support of the wavelet), and periodic extension (assuming that signals can be recovered outside of the original support by periodic extension; de Artigas et al. 2006). Detailed 
features of these border conditions have been examined in previous studies (Su et al. 2011; Nalley et al. 2012). To demonstrate their influence on the forecasting model, all of these border conditions are compared.

By varying the combination of these three factors, this process resulted in a group of 90 different WS models. We established a pool of candidate models consisting of WS models with "good" predictive performance. The forecasts from multiple candidate models were used to produce the ensemble forecasts.

\section{b. Bayesian-based ensemble model}

Choosing a single "best" model (here, the best WS model) for predictive inference and analysis may reject other plausible candidate models (Pokhrel et al. 2013). This could lead to statistical bias and an underestimation of the predictive uncertainty caused by structural differences in the WS model related to varying combinations of the three factors described above. BMA provides a relatively effective solution to this problem by combining forecasts from candidate models while allowing for an assessment of structural uncertainty, generally resulting in more reliable probabilistic predictions (Ajami et al. 2007). The BMA mean (or the BMA predictive mean) could be more advantageous than using a simple model average or the single "best" WS model. More importantly, this approach yields the BMA variance, which measures the uncertainty of the probabilistic predictions (Franz et al. 2010). A brief introduction to the essence of the BMA scheme, as originally described by Raftery et al. (2005), is given below.

In BMA, the probabilistic distribution of a hydrological prediction is the weighted average of the posterior distribution under each candidate model, weighted by their posterior model probabilities. Consider the forecasted variable $y$, the ensemble of all candidate model predictions $\mathbf{f}=\left(f_{1}, f_{2}, \ldots, f_{K}\right)$, and the training data $D$. The $p_{k}\left(y \mid f_{k}, D\right)$ is the posterior distribution of $y$ given model prediction $f_{k}$ and training data $D$. The posterior distribution of the BMA prediction is then represented as

$p\left(y \mid f_{1}, f_{2}, \ldots, f_{K}, D\right)=\sum_{k=1}^{K} p\left(f_{k} \mid D\right) p_{k}\left(y \mid f_{k}, D\right)$,

where $p\left(f_{k} \mid D\right)$ is defined as the posterior probability of model prediction $f_{k}$, also known as the likelihood of model $f_{k}$ being the correct prediction given training data $D$. This term reflects how well candidate model $f_{k}$ fits the training data (Duan et al. 2007). If we denote $\mathbf{w}_{k}=p\left(f_{k} \mid D\right)$, then $\sum_{k=1}^{K} \mathbf{w}_{k}=1$, and they can hence be viewed as weights. According to Raftery et al. (2005) and Duan et al. (2007), the posterior mean and variance of the BMA prediction can be calculated as

$$
\begin{aligned}
E\left(y \mid f_{1}, f_{2}, \ldots, f_{K}, D\right) & =\sum_{k=1}^{K} p\left(f_{k} \mid D\right) E\left[p_{k}\left(y \mid f_{k}, D\right)\right] \\
& =\sum_{k=1}^{K} \mathbf{w}_{k} f_{k}
\end{aligned}
$$

and

$\operatorname{Var}\left(y \mid f_{1}, f_{2}, \ldots, f_{K}, D\right)=\sum_{k=1}^{K} \mathbf{w}_{k}\left(f_{k}-\sum_{i=1}^{K} \mathbf{w}_{i} f_{i}\right)^{2}+\sigma^{2}$,

where $\sigma^{2}$ is the variance of the time series based on one of the model predictions being the best at each time step. Suppose we build a time series that, at each time step, includes the best prediction (closest to the observation) from one of the candidate models $-\sigma^{2}$ represents the variance of this time series with regard to other observations (Ajami et al. 2007). The two terms of the right-hand side of Eq. (10) indicate the between-model variance that presents between-model spread (or ensemble spread) and within-model variance that measures the expected uncertainty conditioned on one of the forecasts being the best at any given time step (Franz et al. 2010). Equation (9) is a better description of predictive uncertainty than that in a non-BMA scheme, which estimates uncertainty based only on the ensemble spread (i.e., only the between-model variance is considered; Raftery et al. 2005; Franz et al. 2010).

When implementing the BMA algorithm, it is usually assumed that the conditional probability $p_{k}\left(y \mid f_{k}, D\right)$ is Gaussian. As a Gaussian distribution cannot represent the highly skewed predictive distribution of streamflow or other hydrological variables dominantly driven by rainfall, this assumption may be inappropriate in the present study. We replace the conditional probability $p_{k}\left(y \mid f_{k}, D\right)$ by implementing the gamma distribution illustrated by Sloughter et al. (2007), although Vrugt and Robinson (2007) showed that the assumption of a Gaussian distribution also works well for streamflow simulations and forecasting in the BMA-based ensemble of different conceptual hydrological models. BMA requires the weights and variance of the individual candidate models to be specified. Following the recommendation of Raftery et al. (2005) and Duan et al. (2007), we use the expectation maximum (EM) algorithm for this purpose. The EM algorithm is easy to implement, computationally efficient, and the maximization step always satisfies the constraint that the weights are positive and sum to 1 (Vrugt and Robinson 
2007). The detailed procedure of applying the EM algorithm is given in Raftery et al. (2005) and Duan et al. (2007). We implement the BMA approach by dynamically updating the weights and variance of the individual candidate models in the ensemble.

\section{c. Performance measures}

Three frequently used statistics in model evaluation are employed to assess the accuracy of different models. They are the coefficient of determination $R^{2}$, root-meansquare error (RMSE), and Nash-Sutcliffe efficiency (NSE; Nash and Sutcliffe 1970; Dawson et al. 2007; Bennett et al. 2013).

The coefficient of determination measures the degree of correlation among the observed and predicted values. It ranges from 0 to 1 , with higher values indicating better model performance, and is expressed as

$$
R^{2}=\frac{\sum_{i=1}^{N}\left(\hat{\mathbf{y}}_{i}-\overline{\mathbf{y}}_{i}\right)^{2}}{\sum_{i=1}^{N}\left(\mathbf{y}_{i}-\overline{\mathbf{y}}_{i}\right)^{2}},
$$

where

$$
\overline{\mathbf{y}}_{i}=\frac{1}{N} \sum_{i=1}^{N} \mathbf{y}_{i} .
$$

Here, $N$ is the number of data points, $\hat{\mathbf{y}}_{i}$ is the predicted value, $\mathbf{y}_{i}$ is the observed value, and $\overline{\mathbf{y}}_{i}$ is the mean of the observed data values for the entire time period.

The RMSE is used to measure the average error magnitude. It is given by

$$
\mathrm{RMSE}=\sqrt{\frac{1}{N} \sum_{i=1}^{N}\left(\mathbf{y}_{i}-\hat{\mathbf{y}}_{i}\right)^{2}},
$$

where the variables are as above. RMSE indicates the discrepancy between the observed and forecasted values. A perfect fit between observed and forecasted values would give an RMSE of 0 .

The NSE indicates how well the plot of the observed values versus the simulated values fits the 1:1 line. NSE ranges from $-\infty$ to 1 , with larger values signifying better model performance. It is calculated as

$$
\mathrm{NSE}=1-\frac{\sum_{i=1}^{N}\left(\mathbf{y}_{i}-\hat{\mathbf{y}}_{i}\right)^{2}}{\sum_{i=1}^{N}\left(\mathbf{y}_{i}-\overline{\mathbf{y}}_{i}\right)^{2}},
$$

with the variables defined as before.
The containing ratio $(\mathrm{CR})$ is used to assess the prediction uncertainty of intervals generated by uncertainty analysis methods. It is defined as the percentage of observations that are covered in the prediction ranges:

$$
\begin{aligned}
\mathrm{CR} & =\frac{\sum_{i=1}^{N} l\left(\mathbf{y}_{i}\right)}{N} \\
\text { with } l\left(\mathbf{y}_{i}\right) & =\left\{\begin{array}{ll}
1, & \mathbf{L}_{i}<\mathbf{y}_{i}<\mathbf{U}_{i} \\
0, & \text { otherwise }
\end{array} .\right.
\end{aligned}
$$

The variables $\mathbf{L}_{i}$ and $\mathbf{U}_{i}$ are the lower and upper ranges, respectively, of the $90 \%$ confidence interval from the BMA prediction (Franz et al. 2010).

\section{WS model assessment and ensemble prediction}

\section{a. Impact of the three factors on overall performance of WS models}

We first evaluated the effectiveness of a variety of WS models. As an example, we consider the 1-day-ahead forecasting at Longchuan to illustrate the effect of these proposed factors on forecasting (see Fig. 3). We found that the forecasting performance of different WS models varied significantly, and not all of them produced reliable forecasts. This highlights the importance of considering the three proposed factors in wavelet-based forecasting. Regarding the appropriate mother wavelet for a certain application, some studies stated that wavelets with a specific time-frequency localization property would enable the forecasting model to capture the underlying trend and short-term variability in a particular daily streamflow time series (Nourani et al. 2011, 2013, 2014). In this case, we found that models using the sym5 wavelet generally provide better forecasts in terms of our performance measures, whereas the highest accuracy (in terms of NSE and RMSE) was obtained using the $\mathrm{db} 4$ wavelet (three decomposition levels in symmetrization extension mode). As illustrated in Fig. 3, increasing the number of decomposition levels diminishes the forecasting accuracy for most wavelets. With respect to the sensitivity of different border extensions, the WS models using symmetrization extension provide higher forecasting accuracy than those using periodic extension for most wavelets (Fig. 3). This can be partly explained by the fact that symmetrization extension has the advantage of avoiding artificial discontinuities near borders, whereas periodic extension could introduce distortions to the wavelet transform because of boundary discontinuities (Lin and Smith 2008; Su et al. 2011). However, it should be noted that periodic extension outperforms symmetrization for a 

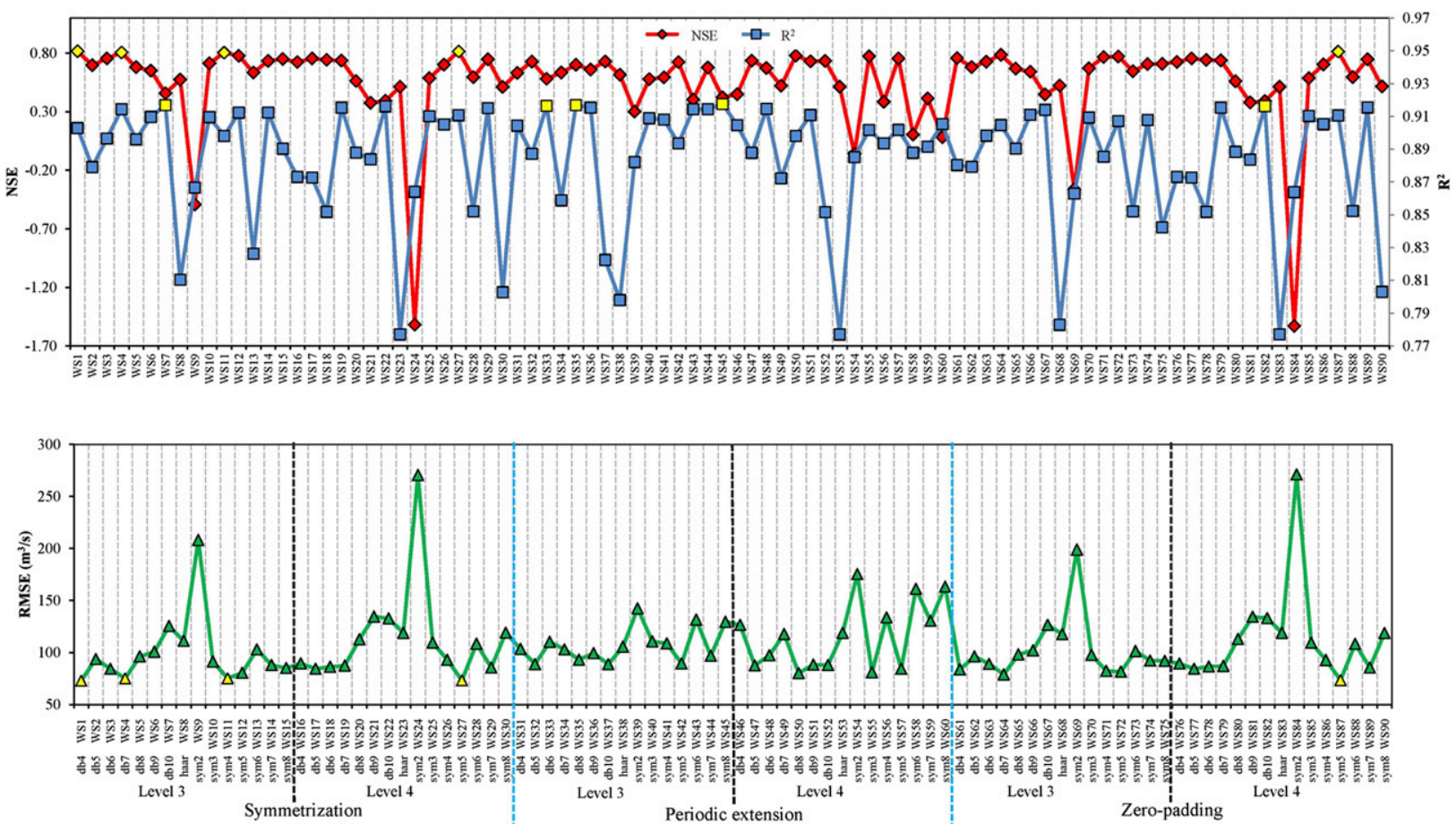

FIG. 3. Performance of the WS models for different mother wavelets, decomposition levels, and border conditions for 1-day-ahead forecasting in the validation period at Longchuan.

few wavelets. This emphasizes that the overall forecasting performance of WS models is not only influenced by border conditions, but also relies strongly on the properties of wavelets and decomposition levels. Moreover, it is interesting that the simple zero-padding extension gives a similar performance to symmetrization, and generally outperforms periodic extension.

\section{$b$. Impact of the proposed factors on the performance of WS models in different streamflow ranges}

Different combinations of the wavelet functions, decomposition levels, and edge effects may perform differently for low, medium, and high streamflows. That is, their ability to capture particular hydrological events could vary. In this section, we examine the performance of each WS model across different streamflow ranges. We first fitted a nonexceedance probability curve of the historical observations (in the validation period) using a gamma distribution (see Fig. S1 in the supplemental material). Thresholds for high, medium, and low flow were then determined. For this study, flows that did not exceed $33.3 \%$ and $66.7 \%$ of the observed flows were used to separate the historical observations into low, medium, and high categories. Figure 4 illustrates the prediction skill of different WS models in the three flow ranges and the whole hydrograph during the validation period in terms of the NSE. Again, the prediction accuracy fluctuates considerably for different wavelet, decomposition level, and border condition combinations. In general, WS models seem to give better predictions for high flows than for low and medium flows, whose NSE scores are mostly negative and produce relatively unreliable forecasts (Fig. 4). We also observed that the pattern of prediction accuracy for high flows generally agrees with the overall performance across the whole hydrograph, whereas the patterns for both low and medium flows are significantly different from those of the overall performance. This suggests that the results from high streamflows could be the dominant contributor to the overall prediction skill in the entire hydrograph. We further compared the performance of the best individual WS models for each flow range and the whole hydrograph using the flow-duration curve (FDC; Vogel and Fennessey 1995), which represents the frequency distribution of streamflow over a specific time period (e.g., daily or monthly). All FDCs are presented as logarithmic plots. As illustrated in Fig. 5, the FDCs generated from the best individual WS models for low, medium, and high flows are more consistent with the observations in their corresponding flow ranges than the WS model that gives the best overall forecasting performance for the whole hydrograph. However, it should be emphasized that this study focuses on improving the overall prediction skill across the whole hydrograph, 


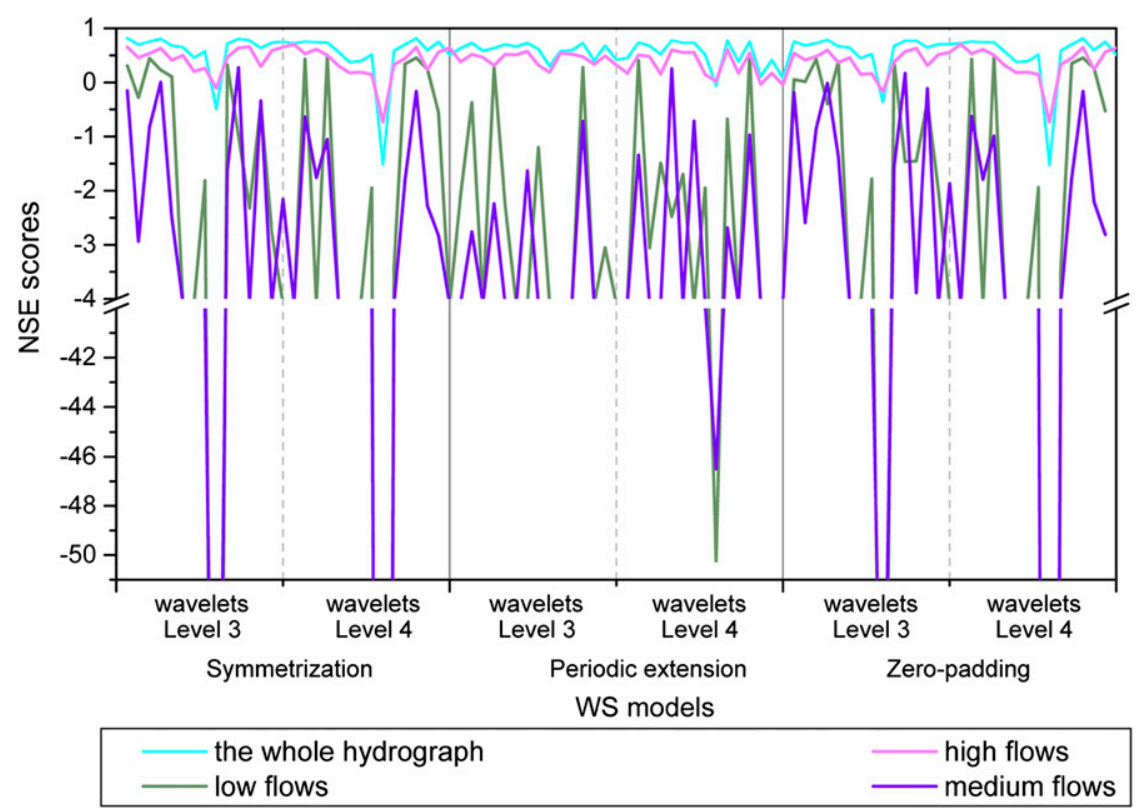

FIG. 4. NSE statistics of different WS models established by varying combinations of three proposed factors in low, medium, and high flow ranges, as well as the whole hydrograph during the validation period. The term "wavelets" indicates the wavelet functions arranged in the order: db4-10, Haar, and sym2-8.

rather than for any particular flow range. Next, we will work with WS models based on their overall forecasting performance across the whole hydrograph.

\section{c. Ensemble prediction}

The use of forecasts generated from the individual model with the best overall prediction skill could be problematic, as this approach excludes other plausible models with similar overall performance and ignores model uncertainty. Therefore, an ensemble prediction (i.e., the BWS model) was generated by combining forecasts from various WS models using the BMA algorithm as introduced in section $4 \mathrm{~b}$. Previous studies using BMA have concluded that the success of ensemble forecasts generally relies on the candidate models performing well individually (Vrugt and Robinson 2007; Pokhrel et al. 2013). Additionally, ensemble forecasts become computationally demanding as more individual members are introduced (Raftery et al. 2005). In light of these, for this study, we consider only the five best WS models (selected according to their NSE scores) as the candidate members. The proposed BWS model produces a final forecast by exploiting the strengths of the selected WS models and provides an assessment of predictive uncertainty associated with the structural deficiencies in individual WS models. In the current study, streamflow prediction was also carried out using ANFIS. Introduced by Jang (1993), ANFIS makes use of the advantages of both ANN and fuzzy system and combines the benefits of both these fields within a single framework (Nourani et al. 2011). A detailed description of ANFIS can be found in Jang (1993). The appropriate

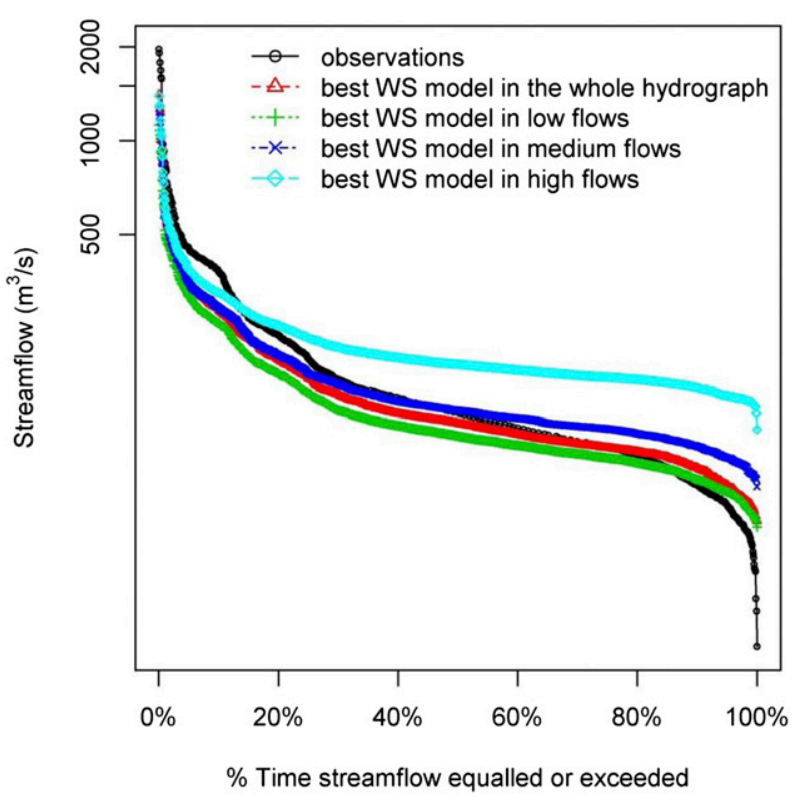

FIG. 5. FDCs for the daily observations and forecasts from the best individual WS models in low, medium, and high flow ranges, as well as the whole hydrograph. 


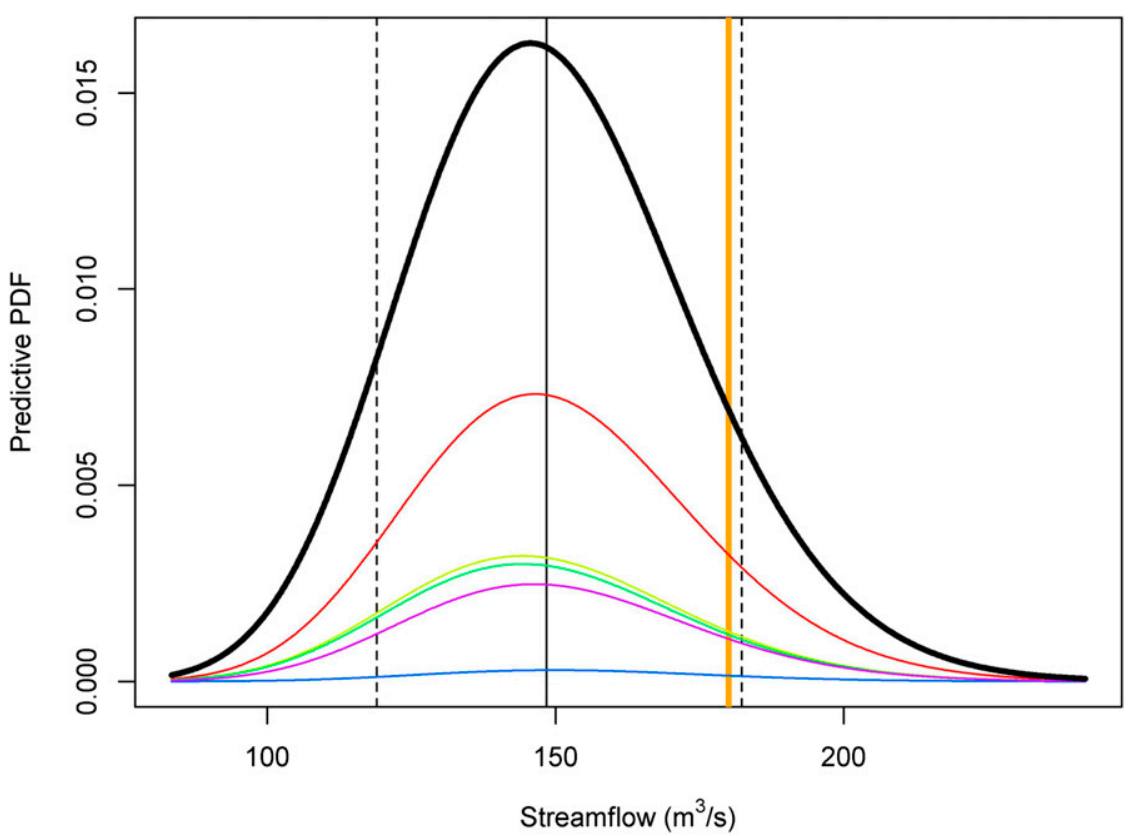

FIG. 6. BMA predictive PDF (thick black curve) and its five conditional PDFs (thin colored curves) for the daily streamflow forecast at Longchuan on $19 \mathrm{Feb} 2008$. The $90 \%$ prediction interval (dotted vertical lines), BMA predictive mean (black vertical line), and verifying observation (orange vertical line) are also shown.

selection of the ANFIS architecture (e.g., the type and number of membership function) is determined by a trial-and-error method (Nourani et al. 2011). We compared the forecasting performance of our BWS model with that of the best WS model and ANFIS.

\section{Results and discussion}

\section{a. Illustration of the BMA-integrated approach}

In this section, we illustrate how the BMA-integrated approach is implemented using a single streamflow forecast from one station at one time. This example considers the 1-day-ahead forecast for 19 February 2008 at Longchuan. We obtained the NSE values of the candidate WS models and the corresponding BMA weights (see Table S2 in the supplemental material). There are significant discrepancies among the candidate members in terms of their weights. It should also be noted that there is no direct relationship between the rank order trends of candidate members' NSE values and weights; the weights also reflect the correlations between forecasts from different members (Raftery et al. 2005; Wöhling and Vrugt 2008). Figure 6 shows the BMA predictive probability density function (PDF), which is the weighted sum of the conditional PDFs of the five individual candidate members. The BMA predictive PDF is unimodal and broader than any of the individual conditional PDFs. It can also be found that the individual WS models contradict one another. The observation falls within the $90 \%$ BMA prediction interval.

\section{b. 1-day-ahead forecasting skill}

The daily streamflow forecasts (1-day-ahead) during the validation period (from 1 January 2008 to 31 December 2011) from ANFIS, the best WS model, and the final BWS model (the expected BMA mean) were compared at the two sites in the form of a hydrograph, as shown in Fig. 7. The $90 \%$ prediction uncertainty intervals and associated CRs for the occurrence of observations within the $90 \%$ confidence intervals are also included in Fig. 7. Although it is difficult to visually determine the best prediction model from Fig. 7, the BMA-based probabilistic forecasting is clearly more informative (e.g., in providing the prediction uncertainty) than others (ANFIS and the best WS model). This also highlights the benefit of the BMA-integrated approach over the individual model approach. As can be seen, about $88.2 \%$ (CR value) of all observations are covered by the $90 \%$ confidence bounds at the Longchuan site (Fig. 7a). At Yuecheng (Fig. 7b), the coverage is at $88.3 \%$, and the observations were well captured within the given confidence bounds. Moreover, Fig. 8 compares the observed FDCs with those predicted by the BWS, the best single-member WS model, and 


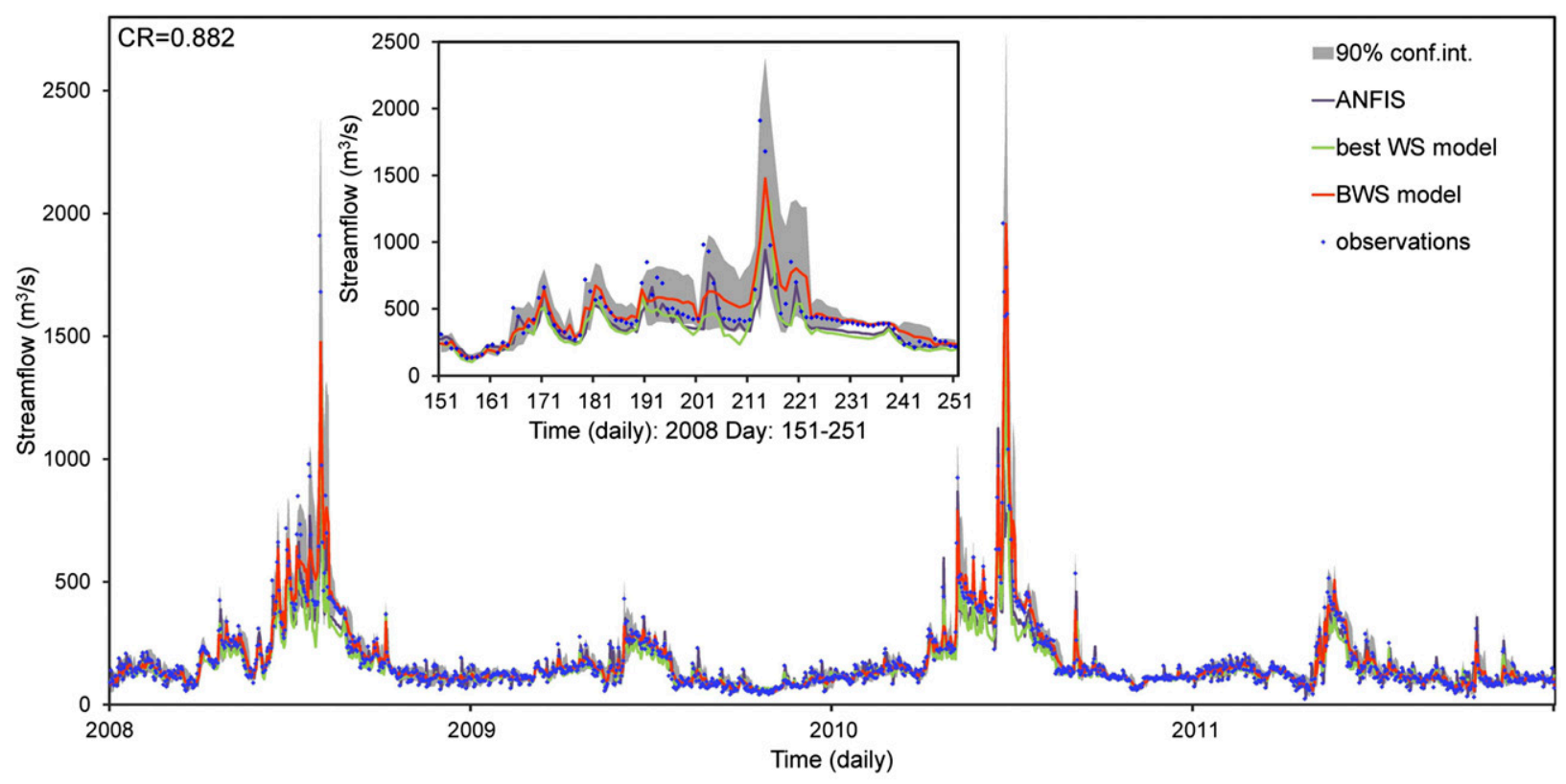

(a)

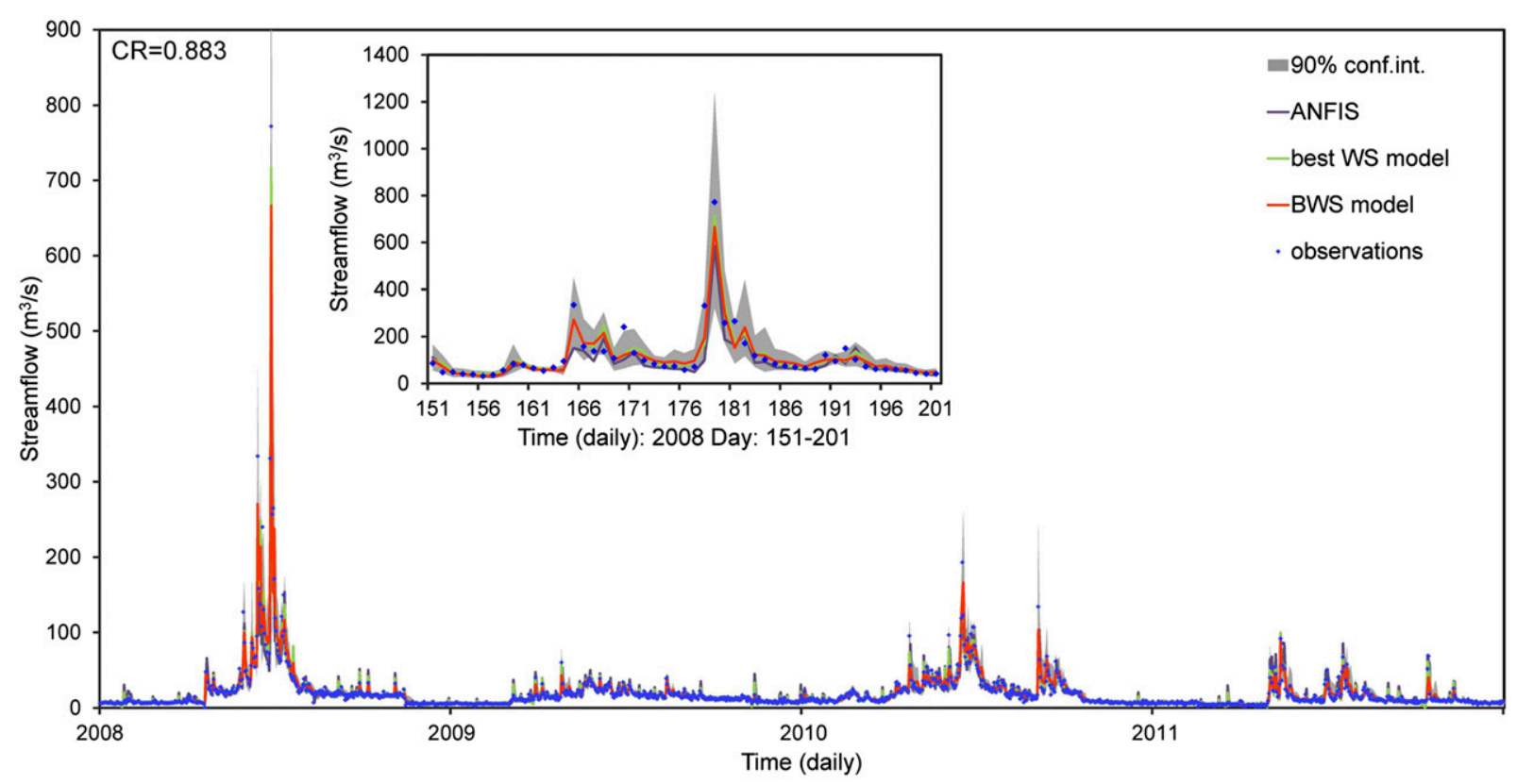

(b)

FIG. 7. Time series plots of observed daily streamflows and forecasts in validation period (from 1 Jan 2008 to 31 Dec 2011) using ANFIS, the best WS model, and the BWS model with $90 \%$ uncertainty prediction intervals and CR for 1-day-ahead forecasting at (a) Longchuan and (b) Yuecheng.

ANFIS for Longchuan and Yuecheng. For the Longchuan site, the predicted FDCs from the BWS model and ANFIS show better agreement with the observed FDC than the best single-member WS model. At
Yuecheng, interestingly, all predicted FDCs seem to track the observed FDC well, while the BWS model still exhibits better consistency than the single WS model. The disparity in prediction skill for 1-day-ahead 


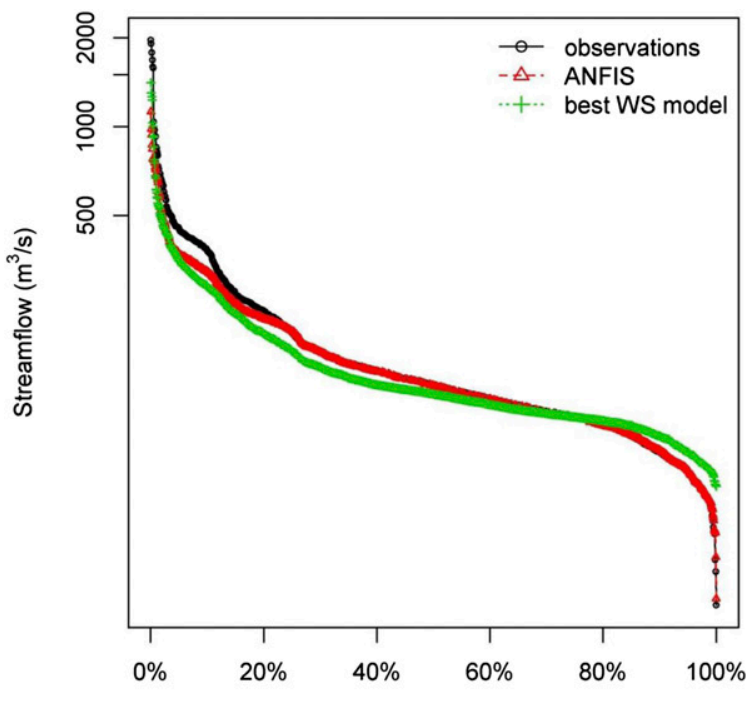

$\%$ Time streamflow equalled or exceeded

(a)

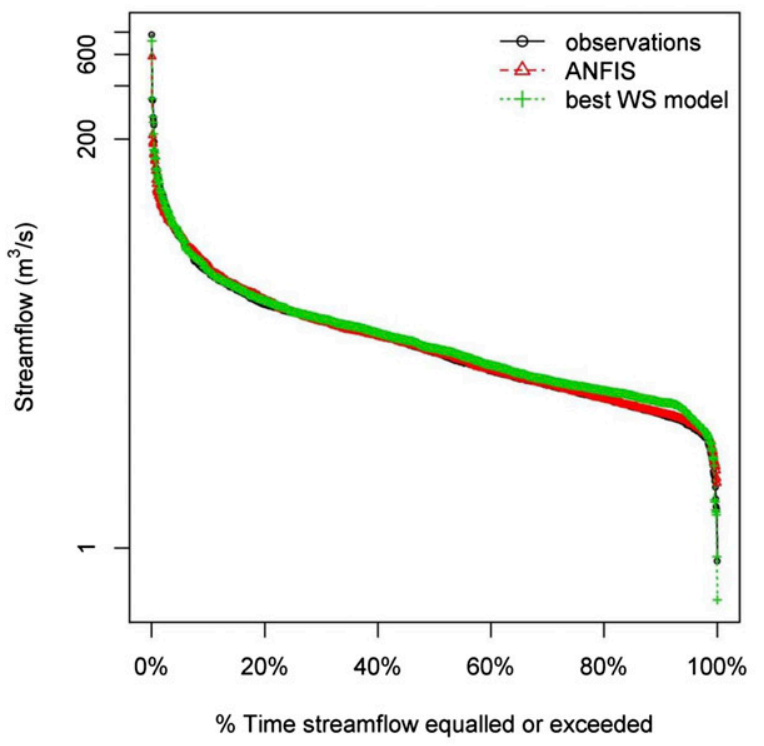

(c)

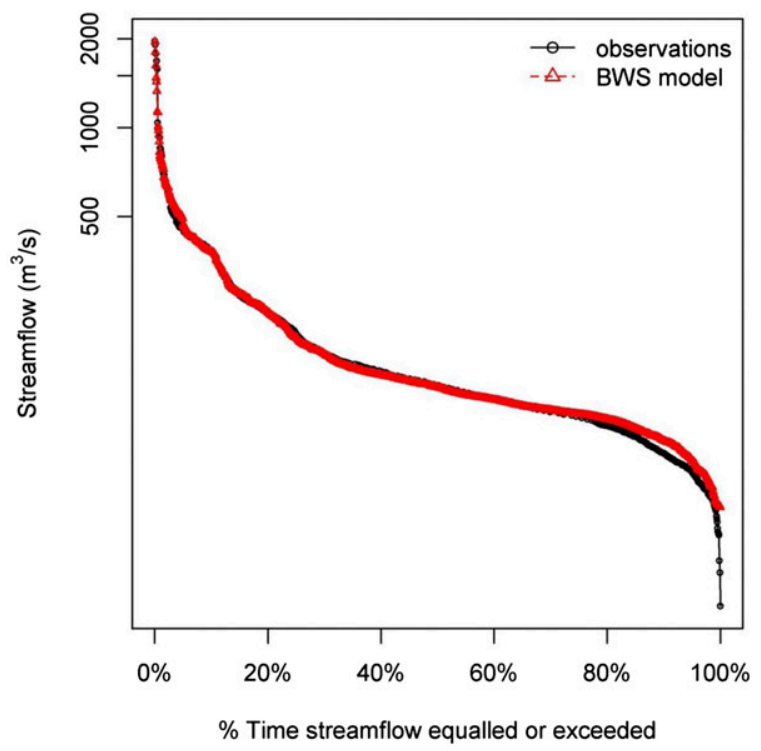

(b)

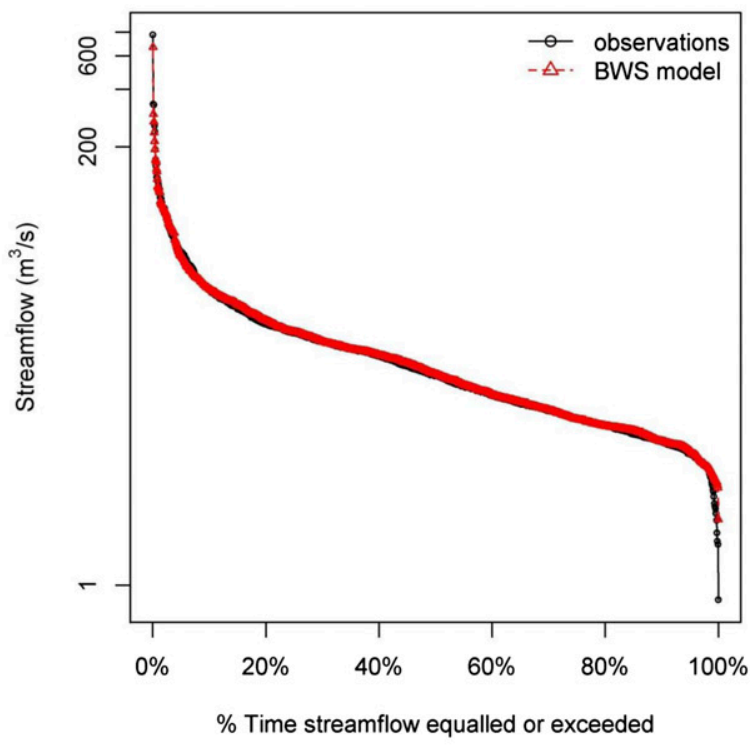

(d)

FIG. 8. FDCs for the daily observations and forecasts from ANFIS, the best WS model, and the BWS model during the validation period at (a),(b) Longchuan and (c),(d) Yuecheng.

forecasting is well illustrated by the quantitative statistics given in Table 1. It is apparent that the proposed BWS model is generally superior, to some extent, to ANFIS and the best single-member WS model at both sites. Overall, with regards to 1-day-ahead forecasting, the BWS model has the ability to provide more accurate and reliable predictions than the best single-member WS model as well as ANFIS.

\section{c. 1-month-ahead forecasting skill}

A similar approach to the above was applied for monthly streamflow forecasting. In this instance, we 
TABLE 1. Summary statistics of ANFIS, best WS model, and BWS model for 1-day-ahead and 1-month-ahead forecasting in the validation period at Longchuan and Yuecheng.

\begin{tabular}{|c|c|c|c|c|c|c|}
\hline \multirow[b]{2}{*}{ Model } & \multicolumn{3}{|c|}{ Longchuan } & \multicolumn{3}{|c|}{ Yuecheng } \\
\hline & NSE & $\operatorname{RMSE}\left(\mathrm{m}^{3} \mathrm{~s}^{-1}\right)$ & $R^{2}$ & NSE & $\operatorname{RMSE}\left(\mathrm{m}^{3} \mathrm{~s}^{-1}\right)$ & $R^{2}$ \\
\hline \multicolumn{7}{|l|}{ 1-day-ahead forecasting } \\
\hline ANFIS & 0.736 & 87.557 & 0.788 & 0.827 & 13.262 & 0.843 \\
\hline Best WS model & 0.817 & 73.001 & 0.903 & 0.910 & 9.571 & 0.910 \\
\hline BWS model & 0.901 & 53.585 & 0.901 & 0.919 & 9.063 & 0.922 \\
\hline \multicolumn{7}{|l|}{ 1-month-ahead forecasting } \\
\hline ANFIS & 0.394 & 105.172 & 0.412 & 0.195 & 19.657 & 0.205 \\
\hline Best WS model & 0.787 & 62.327 & 0.797 & 0.819 & 9.333 & 0.827 \\
\hline BWS model & 0.863 & 50.033 & 0.868 & 0.949 & 4.960 & 0.950 \\
\hline BWS model (no climate indices input) & 0.861 & 50.296 & 0.866 & 0.923 & 6.077 & 0.925 \\
\hline
\end{tabular}

used monthly climate indices (i.e., ENSO and IOD) as the additional predictors. Again, we obtained a total of 90 single WS models with individual performance statistics (NSE, RMSE, and $R^{2}$ ) in the validation period (results not shown here for reasons of brevity). There was a mix of high and low accuracy in the various WS models using different combinations of wavelets, decomposition levels, and border conditions. Similar to the daily ensemble forecasting, the five WS models with the best NSE scores were used to build the pool of candidate members for monthly ensemble predictions. We then combined the benefits of forecasts from candidate WS models through BMA. A comparison of the BWS model (with $90 \%$ prediction uncertainty intervals), the best WS model, and ANFIS for 1-month-ahead forecasting during the validation period (from January 2008 to December 2011) is shown in the hydrographs of Fig. 9a (Longchuan) and Fig. 9b (Yuecheng). For both sites, the ensemble predictions are clearly more consistent with the observations than the predictions generated from the best singlemember WS model and ANFIS. According to the CR

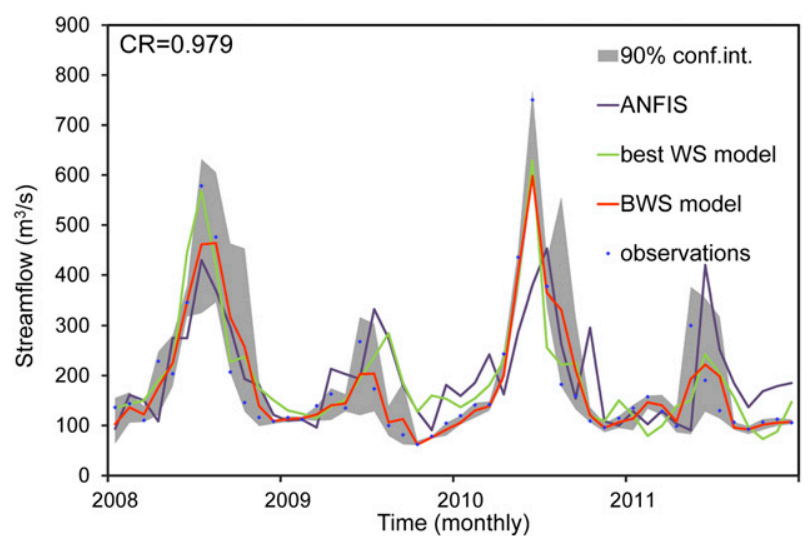

(a) values, the $90 \%$ prediction uncertainty ranges derived for the ensemble model have a high coverage (exceeding $97 \%$ for both sites). The summary statistics of the two models for 1-month-ahead forecasting in the validation period are given in Table 1. At both sites, the BWS model seems to outperform others, demonstrating once again the superiority of applying the BWS model. With regard to the FDCs, the predicted streamflows from the ensemble model generally performed better than the single model and ANFIS for each site (Fig. 10).

\section{d. Forecasting skill of longer lead times}

We further explored the forecasting ability of the proposed BWS model for streamflow forecasting at longer lead times, that is, $2-7$ days for daily forecasting and 2-3 months for monthly forecasting. Again, the candidate members consist of the best five WS models in terms of their NSE scores. The performance of ANFIS, the best single-member WS model, and the BWS model for forecasting lead times of 2-7 days at the two sites is illustrated in Fig. 11. As shown, the BWS model generally

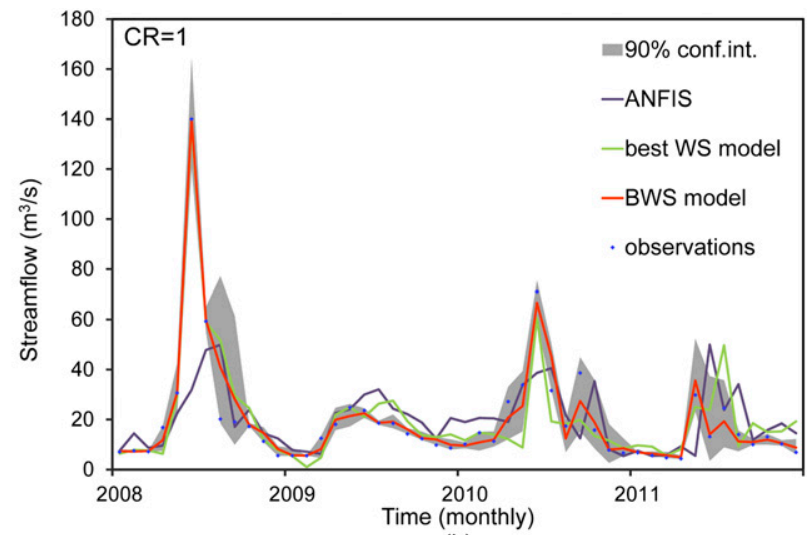

(b)

FIG. 9. Time series plots of observed monthly streamflows and forecasts in validation period (from January 2008 to December 2011) using ANFIS, the best WS model, and the BWS model with $90 \%$ uncertainty prediction intervals and CR for 1-month-ahead forecasting at (a) Longchuan and (b) Yuecheng. 


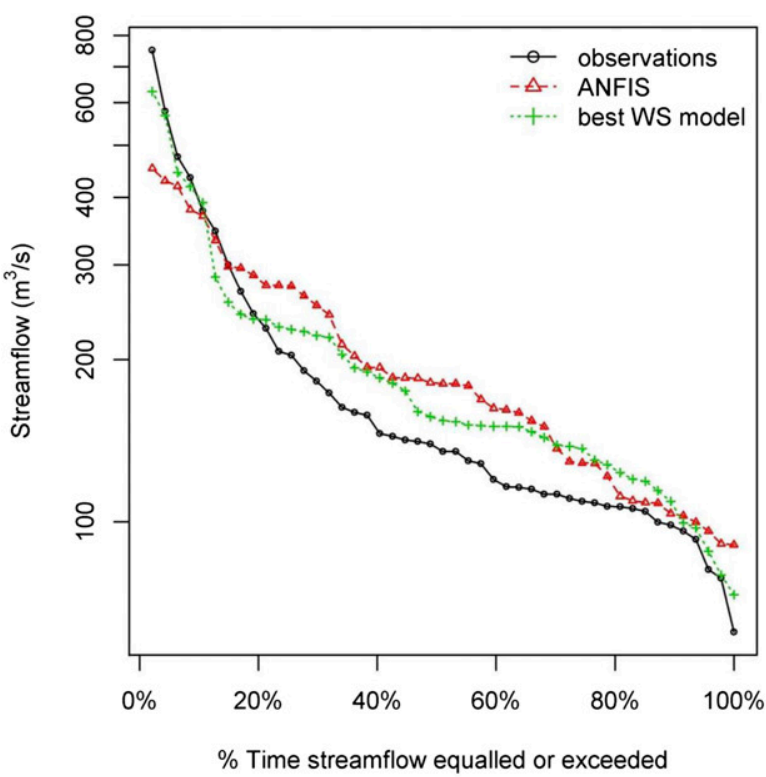

(a)

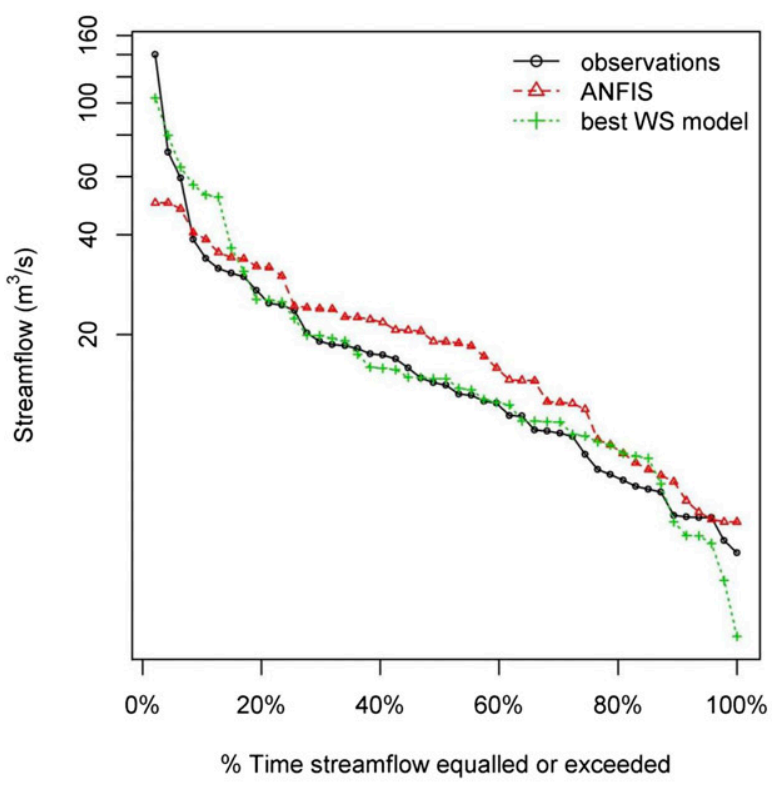

(c)

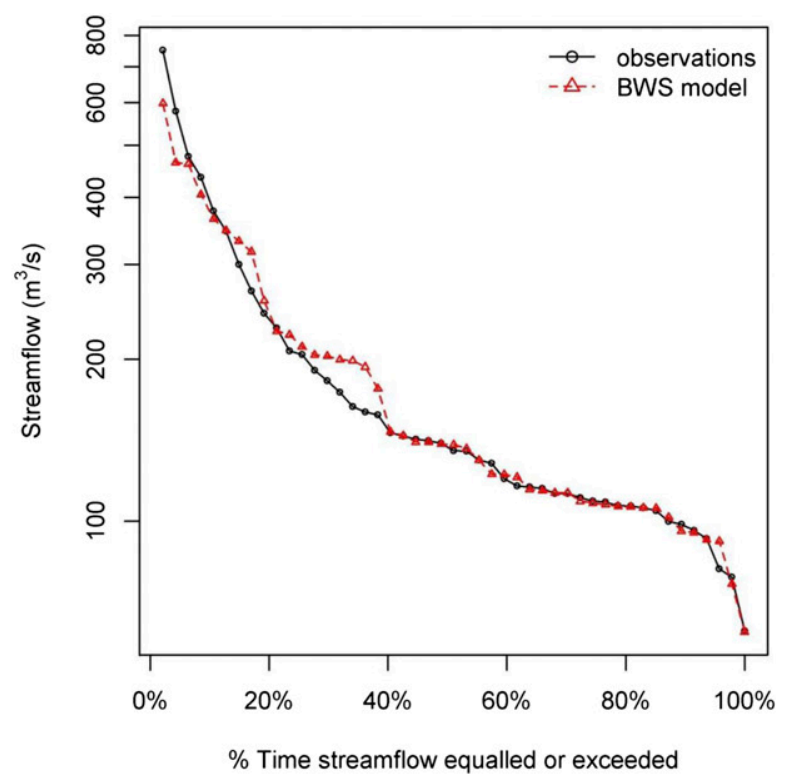

(b)

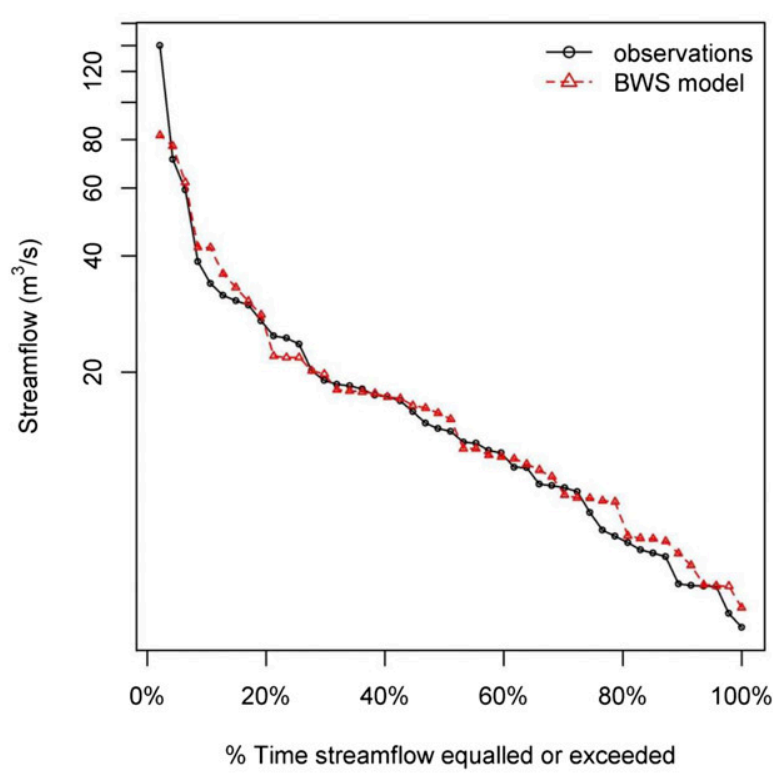

(d)

FIG. 10. FDCs for the monthly observations and forecasts from ANFIS, the best WS model, and the BWS model during the validation period at (a),(b) Longchuan and (c),(d) Yuecheng.

outperformed the single WS model and ANFIS in terms of the statistics. Even for lead times of 5-7 days, the advantage of the BMA-based model is pronounced at both sites (NSE scores greater than 0.7).

Monthly streamflow forecasting for lead times of 2 3 months was also carried out using ANFIS, the best single-member WS model, and the ensemble model. A comparison of the monthly forecasting skill of different methods for the two sites is presented in Fig. 12. As expected, the BMA-based model consistently resulted in more accurate and reliable forecasts. Moreover, we can also note that the model forecasting skill differs 


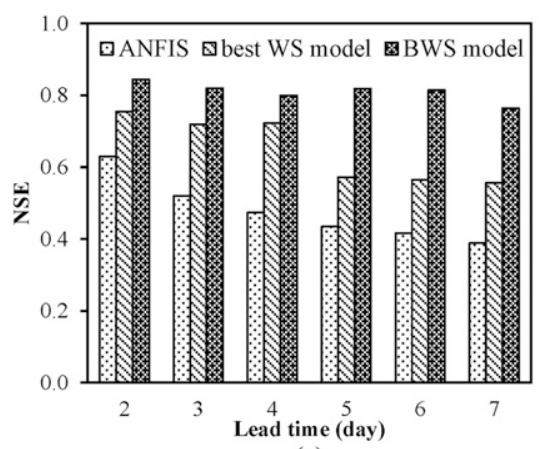

(a)

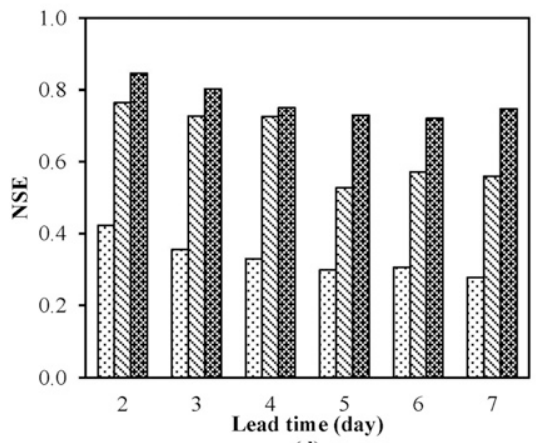

(d)

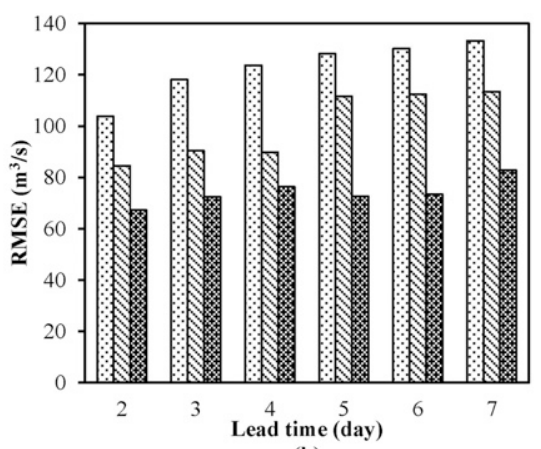

(b)

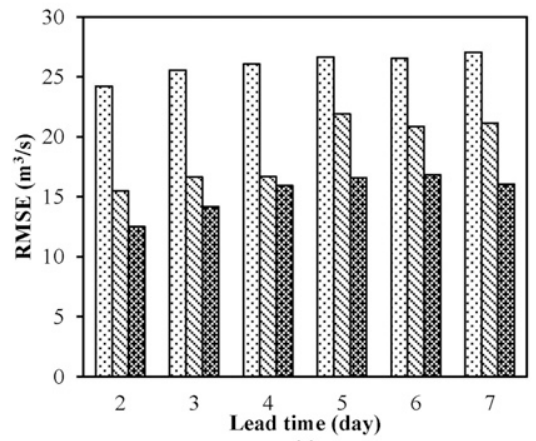

(e)

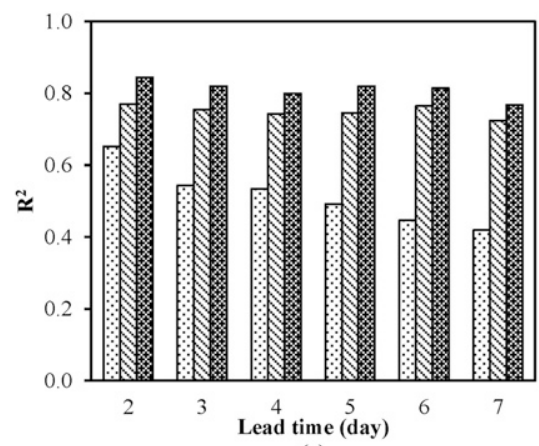

(c)

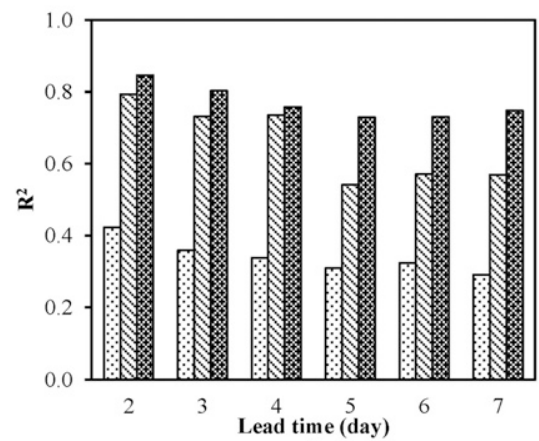

(f)

FIG. 11. Performance of ANFIS, the best WS model, and the BWS model for 2-7-days-ahead forecasting in the validation period at (a)-(c) Longchuan and (d)-(f) Yuecheng.

between the two sites for both daily and monthly forecasting. This suggests that the predictability is also reliant on the natural variability of the streamflow time series, which could be strongly affected by the factors including local micrometeorological conditions (e.g., rainfall and soil moisture) and topographic features in a certain subclimate zone (Dhanya and Kumar 2011). Overall, the proposed BWS model resulted in a more skillful prediction for both daily and monthly streamflow forecasting at longer time leads, indicating that the application of Bayesian techniques is able to provide useful insights into the limitations of the WS model and can thus develop a suitable structure and complexity for an ensemble model.

Moreover, we further assessed whether the inclusion of climate indices as predictors improves the prediction accuracy. The accuracy of the BWS models without considering climate indices for monthly streamflow forecasts at the two sites is presented in Table 1 and Fig. 12. The results show the use of climate indices provides additional accuracy to some extent.

\section{e. Skill assessment over various validation periods}

In this section, we examine how the BMA scheme performs across different validation periods for the observed hydrograph. We take the example of the 1-day-ahead streamflow prediction at Longchuan. The prediction skill of the BWS model was separately assessed for each year of the entire validation period (2008-11). Table 2 presents performance measures across four individual validation periods regarding 1-day-ahead streamflow prediction at Longchuan. The average BMA weights of individual candidate WS models for these validation periods are also listed. For each validation period, the BWS model produced reliable forecasts in terms of the NSE and $R^{2}$. It is interesting to note that the BMA weights of individual candidate WS models differ between the four validation periods. However, we can see that the WS model (WS1), which uses the db4 wavelet and three decomposition levels in symmetrization extension mode, generally receives the highest weight for each validation period.

\section{f. Sensitivity of selecting candidate models based on different statistics and the variability of candidate models for different cases}

The results presented above show that the ensemble BWS model gives consistent improvements in streamflow prediction skill over the best single-member WS model and ANFIS. We now explore the impact of selecting candidate WS models with respect to different performance statistics on the overall prediction skill of 


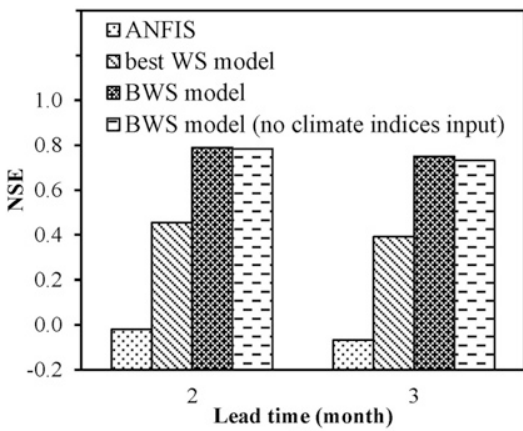

(a)

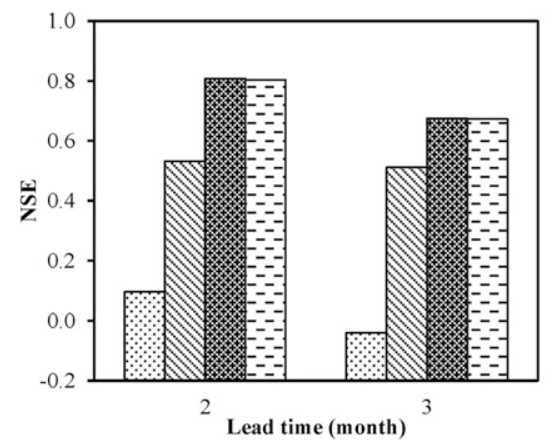

(d)

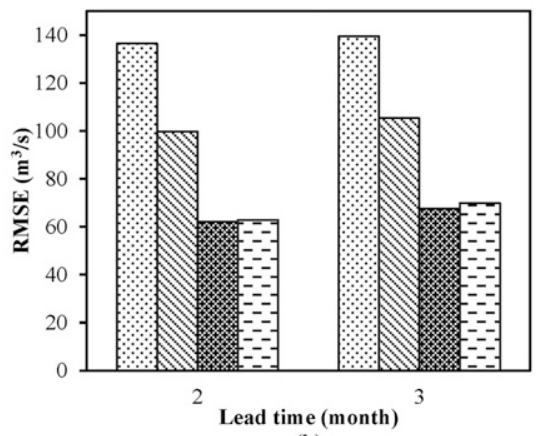

(b)

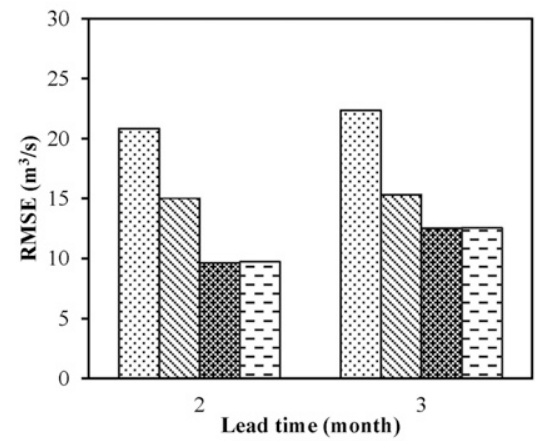

(e)

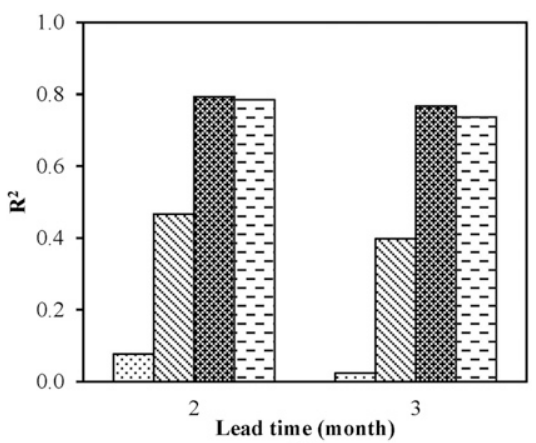

(c)

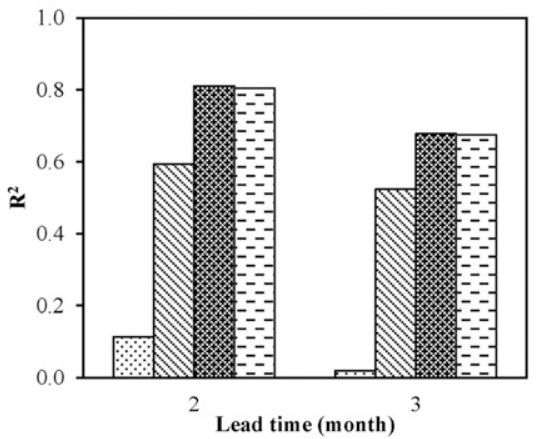

(f)

FIG. 12. Performance of ANFIS, the best WS model, and the BWS model for 2-3-months-ahead forecasting in the validation period at (a)-(c) Longchuan and (d)-(f) Yuecheng.

the BWS model. To illustrate this, we again use the example of 1-day-ahead streamflow prediction at Longchuan. Instead of the NSE, the five candidate models were chosen according to their $R^{2}$ scores. We then combined multiple candidate models using BMA (denoted as $\mathrm{BWS}_{R^{2}}$ ). Compared to the original BWS model (denoted as $\mathrm{BWS}_{\mathrm{NSE}}$ ), the performance of $\mathrm{BWS}_{R^{2}}$ is significantly inferior (Table 3). A similar test concerning the RMSE scores $\left(\mathrm{BWS}_{\mathrm{RMSE}}\right)$ was also applied. The performance of BWS $_{\text {RMSE }}$ model was found to be similar to that when the NSE criterion was applied. We compared the corresponding five candidate WS models regarding the different measurement criteria (see the yellow marker points in Fig. 3). It can be seen that the candidate models selected by NSE scores agree with those given by RMSE. As for $R^{2}$ criterion, although the chosen WS models have high $R^{2}$ scores, their NSE and RMSE scores are very low. This is because $R^{2}$ focuses on measuring the degree of correlation among the observed and predicted values, whereas NSE assesses how well the plot of the observed values versus the simulated values fits the 1:1 line, and RMSE measures the average error magnitude (see the equations in section $4 \mathrm{c}$ ). The WS model with the highest $R^{2}$ score (high correlation) does not necessarily produce predicted values that are close to the observed values, nor is the average error magnitude low. By comparison, both $\mathrm{BWS}_{\mathrm{NSE}}$ and $\mathrm{BWS}_{\mathrm{RMSE}}$ exhibit superior prediction accuracy and are thus more competitive than the $\mathrm{BWS}_{R^{2}}$ model.

Additionally, it might be interesting to investigate the variability of five candidate WS models (selected by the

TABLE 2. Summary statistics of the 1-day-ahead streamflow prediction using the BWS models for four independent validation periods at Longchuan. The average BMA weights of candidate WS models for each validation period are also listed. The characteristics of individual WS models refer to Fig. 3.

\begin{tabular}{|c|c|c|c|c|c|c|c|c|}
\hline \multirow[b]{2}{*}{ Validation period } & \multirow[b]{2}{*}{ NSE } & \multirow[b]{2}{*}{$\operatorname{RMSE}\left(\mathrm{m}^{3} \mathrm{~s}^{-1}\right)$} & \multirow[b]{2}{*}{$R^{2}$} & \multicolumn{5}{|c|}{ Weights } \\
\hline & & & & WS1 & WS27 & WS87 & WS4 & WS11 \\
\hline 2008 & 0.854 & 75.652 & 0.855 & 0.380 & 0.155 & 0.106 & 0.225 & 0.134 \\
\hline 2009 & 0.890 & 19.232 & 0.930 & 0.398 & 0.204 & 0.116 & 0.179 & 0.102 \\
\hline 2010 & 0.921 & 66.853 & 0.922 & 0.377 & 0.204 & 0.174 & 0.160 & 0.086 \\
\hline 2011 & 0.859 & 27.925 & 0.859 & 0.275 & 0.267 & 0.174 & 0.171 & 0.114 \\
\hline
\end{tabular}


TABLE 3. Summary statistics of the $\mathrm{BWS}_{\mathrm{NSE}}, \mathrm{BWS}_{R^{2}}$, and $B W S_{\text {RMSE }}$ models for 1-day-ahead forecasting in the validation period at Longchuan.

\begin{tabular}{lcccc}
\hline \hline & NSE & RMSE $\left(\mathrm{m}^{3} \mathrm{~s}^{-1}\right)$ & $R^{2}$ & $\mathrm{CR}$ \\
\hline $\mathrm{BWS}_{\text {NSE }}$ & 0.901 & 53.585 & 0.901 & 0.882 \\
$\mathrm{BWS}_{R^{2}}$ & 0.502 & 120.317 & 0.545 & 0.635 \\
$\mathrm{BWS}_{\text {RMSE }}$ & 0.901 & 53.585 & 0.901 & 0.882 \\
\hline
\end{tabular}

NSE criterion) for different cases, for example, time series with various scales (daily and monthly) and varying lead times (e.g., 1-7 days and 1-3 months). As an illustration, we take the example of daily and monthly forecasts with different lead times at Longchuan. In general, the candidate WS models vary in each case (Table 4). The difference between daily and monthly forecasts is understandable, as the daily and monthly time series have various physical structures and data lengths, and different mother wavelets have individual capacities for capturing effective features in different kinds of time series (Maheswaran and Khosa 2012a; Nalley et al. 2012; Nourani et al. 2014). In the cases of daily streamflow forecasts with different lead times, we also noticed that the candidate WS models vary from 1-day-ahead to 7-days-ahead predictions. A similar situation exists in the monthly forecasts. This suggests that a certain WS model (with particular mother wavelet, decomposition level, and border condition) producing highly accurate one-step-ahead predictions is not guaranteed to exhibit the same accuracy for other lead times. This confirms that there is no universal combination of mother wavelet, decomposition level, and border conditions that fits all forecast cases and highlights again the importance of seriously considering these three key issues when using the wavelet-based prediction model. More details concerning the variability of candidate WS models for forecasts with different lead times will be investigated in future work.

\section{Summary and conclusions}

Accurate and reliable streamflow forecasting is important for effective and sustainable water resources planning and management. In this study, local meteohydrological observations, as well as climate indices (ENSO and IOD), were used as potential predictors for one- and multistep-ahead streamflow forecasting at two sites in Dongjiang basin (southern China) using an ensemble approach. The ensemble approach was accomplished via a two-step strategy. First, we evaluated the performance of a coupled wavelet-SVR model (i.e., the WS model) for streamflow prediction by considering the mother wavelets, decomposition levels, and edge effects of the wavelet decomposition phase. We found that the difference in accuracy produced by the various combinations of these key factors was noticeable. It should thus be emphasized that, when applying waveletbased forecasting, it is necessary to carefully consider these factors, rather than choosing them based on personal preferences. Second, we combined forecasts from the multiple candidate WS models with high forecasting accuracy using BMA, thus combining the strengths of the forecasts made by different candidate models.

The results show that BMA successfully combines the strengths of forecasts generated by single WS models with "good" performance, and the proposed ensemble model (i.e., the BWS model) was consistently superior to the traditional best single-member WS model and ANFIS for daily (lead times of 1-7 days) and monthly (lead times of 1-3 months) streamflow forecasting. The use of a BMA-integrated method in this study also allows the development of confidence intervals to assess the forecasting uncertainty. Improvements in forecast accuracy were observed at both sites considered in the current study. This indicates that the proposed BMAintegrated wavelet-SVR strategy is promising in terms of improving the overall prediction performance of daily and monthly streamflow estimates. Moreover, we examined the impact on overall prediction skill of choosing candidate members according to different measures. The current work provides a suitable reference for improving hydrological forecasting when using waveletbased machine learning approaches and also has forecasting potential using other time series (e.g., climate variables, groundwater, and urban water demand). The presented approach could be implemented for

TABLE 4. Five best WS models for 1-7-days-ahead and 2-3-months-ahead forecasting at Longchuan. The characteristics of individual WS models refer to Fig. 3 .

\begin{tabular}{|c|c|c|c|c|c|c|c|c|c|c|}
\hline Rank & 1 day & 2 days & 3 days & 4 days & 5 days & 6 days & 7 days & 1 month & 2 months & 3 months \\
\hline 1 & WS1 & WS40 & WS9 & WS41 & WS63 & WS75 & WS75 & WS2 & WS76 & WS27 \\
\hline 2 & WS27 & WS14 & WS43 & WS9 & WS58 & WS43 & WS65 & WS32 & WS16 & WS16 \\
\hline 3 & WS87 & WS78 & WS69 & WS45 & WS49 & WS49 & WS85 & WS62 & WS20 & WS37 \\
\hline 4 & WS4 & WS18 & WS41 & WS69 & WS74 & WS69 & WS25 & WS47 & WS22 & WS20 \\
\hline 5 & WS11 & WS55 & WS45 & WS84 & WS60 & WS28 & WS22 & WS17 & WS50 & WS47 \\
\hline
\end{tabular}


hydrological forecasting in other catchments around the world. For instance, we also tested the performance of the BWS model by forecasting the streamflow in a catchment located in Michigan, United States (see Table $\mathrm{S} 3$ in the supplemental material). In this case, the BWS model yielded more reliable forecasts than the best single-member WS model and ANFIS. Future study will include an explicit evaluation of the influence of input errors, the WS model structure, and the parameters with a total uncertainty assessment. Additionally, the current study combined a limited number of candidate WS models. Some outstanding questions concern the variability of candidate WS models (e.g., different time scales, lead times, watersheds) and how the number of candidate models impacts on the prediction skill of the BWS model. These issues are worth exploring in further work.

Acknowledgments. The study was financially supported by the State Forestry Administration Public Benefit Research Foundation of China (201204104, 201104005), the key program of National Natural Science Funds (4143000213), and State Forestry Administration 948 Innovative Significant Project of China (2011-76). Special appreciation is given to the Guangdong Hydrological Bureau (China) for providing the streamflow and rainfall data. We thank Dr. Tobias Törnros for the helpful advice.

\section{REFERENCES}

Adamowski, J., and K. Sun, 2010: Development of a coupled wavelet transform and neural network method for flow forecasting of non-perennial rivers in semi-arid watersheds. J. Hydrol., 390, 85-91, doi:10.1016/j.jhydrol.2010.06.033.

Ajami, N. K., Q. Duan, and S. Sorooshian, 2007: An integrated hydrologic Bayesian multimodel combination framework: Confronting input, parameter, and model structural uncertainty in hydrologic prediction. Water Resour. Res., 43 W01403, doi:10.1029/2005WR004745.

Alexandersson, H., 1986: A homogeneity test applied to precipitation data. J. Climatol., 6, 661-675, doi:10.1002/ joc.3370060607.

Azamathulla, H. M., and F. C. Wu, 2011: Support vector machine approach for longitudinal dispersion coefficients in natural streams. Appl. Soft Comput., 11, 2902-2905, doi:10.1016/ j.asoc.2010.11.026.

Bartels, R., 1982: The rank version of von Neumann's ratio test for randomness. J. Amer. Stat. Assoc., 77, 40-46, doi:10.1080/ 01621459.1982.10477764.

Bennett, N. D., and Coauthors, 2013: Characterising performance of environmental models. Environ. Modell. Software, 40,1-20, doi:10.1016/j.envsoft.2012.09.011.

Bray, M., and D. Han, 2004: Identification of support vector machines for runoff modeling. J. Hydroinform., 6, 265-280.

Chang, C. C., and C. J. Lin, 2001: LIBSVM: A library for support vector machines. Accessed 10 January 2015. [Available online at http://www.csie.ntu.edu.tw/ cjlin/libsvm/.]
Chen, Y. D., Q. Zhang, X. Lu, S. R. Zhang, and Z. X. Zhang, 2011: Precipitation variability (1956-2002) in the Dongjiang River (Zhujiang River basin, China) and associated large-scale circulation. Quat. Int., 244, 130-137, doi:10.1016/j.quaint.2010.08.013.

Chou, C. M., 2007: Applying multi-resolution analysis to differential hydrological grey models with dual series. J. Hydrol., 332, 174-186, doi:10.1016/j.jhydrol.2006.06.031.

Daubechies, I., 1990: The wavelet transform, time-frequency localization and signal analysis. IEEE Trans. Inf. Theory, 36, 961-1005, doi:10.1109/18.57199.

Dawson, C. W., R. Abrahart, and L. See, 2007: HydroTest: A webbased toolbox of evaluation metrics for the standardised assessment of hydrological forecasts. Environ. Modell. Software, 22, 1034-1052, doi:10.1016/j.envsoft.2006.06.008.

de Artigas, M. Z., A. G. Elias, and P. F. de Campra, 2006: Discrete wavelet analysis to assess long-term trends in geomagnetic activity. Phys. Chem. Earth, 31, 77-80, doi:10.1016/ j.pce.2005.03.009.

Dhanya, C. T., and D. N. Kumar, 2011: Predictive uncertainty of chaotic daily streamflow using ensemble wavelet networks approach. Water Resour. Res., 47, W06507, doi:10.1029/ 2010WR010173.

Dibike, Y. B., S. Velickov, D. P. Solomatine, and M. B. Abbott, 2001: Model induction with support vector machines: introduction and applications. J. Comput. Civil Eng., 15, 208216, doi:10.1061/(ASCE)0887-3801(2001)15:3(208).

Drucker, H., C. J. C. Burges, L. Kaufman, A. Smola, and V. Vapnik, 1997: Support vector regression machines. Advances in Neural Information Processing Systems 9, M. Mozer, M. I. Jordan, and T. Petsche, Eds., MIT Press, 281-287.

Duan, Q., S. Sorooshian, and V. K. Gupta, 1992: Effective and efficient global optimization for conceptual rainfall-runoff models. Water Resour. Res., 28, 265-284, doi:10.1029/91WR02985.

— N. K. Ajami, X. Gao, and S. Sorooshian, 2007: Multi-model ensemble hydrologic prediction using Bayesian model averaging. Adv. Water Resour., 30, 1371-1386, doi:10.1016/ j.advwatres.2006.11.014.

Franz, K. J., P. Butcher, and N. K. Ajami, 2010: Addressing snow model uncertainty for hydrologic prediction. Adv. Water Resour., 33, 820-832, doi:10.1016/j.advwatres.2010.05.004.

Granger, C. W. J., and R. Ramanathan, 1984: Improved methods of combining forecast accuracy. J. Forecasting, 3, 197-204, doi:10.1002/for.3980030207.

Hoeting, J. A., D. Madigan, A. E. Raftery, and C. T. Volinsky, 1999: Bayesian model averaging: A tutorial. Stat. Sci. 14, 382417, doi:10.1214/ss/1009212519.

Hsu, C. W., C. C. Chang, and C. J. Lin, 2010: A practical guide to support vector classification. Accessed 10 January 2015. [Available online at http://www.csie.ntu.edu.tw/ cjlin/papers/ guide/guide.pdf.]

Hsu, K., H. Moradkhani, and S. Sorooshian, 2009: A sequential Bayesian approach for hydrologic model selection and prediction. Water Resour. Res., 45, W00B12, doi:10.1029/2008WR006824.

JAMSTEC, 2012: Indian Ocean dipole mode dataset. Accessed 30 June 2015. [Available online at http://www.jamstec.go.jp/ frcgc/research/d1/iod/.]

Jang, J. S. R., 1993: ANFIS: Adaptive-network-based fuzzy inference system. IEEE Trans. Syst. Man Cybern., 23, 665-685, doi:10.1109/21.256541.

Kao, L. J., C. C. Chiu, C. J. Lu, and J. L. Yang, 2013: Integration of nonlinear independent component analysis and support vector regression for stock price forecasting. Neurocomputing, 99, 534-542, doi:10.1016/j.neucom.2012.06.037. 
Kim, G., and A. P. Barros, 2001: Quantitative flood forecasting using multisensor data and neural networks. J. Hydrol., 246, 45-62, doi:10.1016/S0022-1694(01)00353-5.

Kisi, O., and M. Cimen, 2011: A wavelet-support vector machine conjunction model for monthly streamflow forecasting. J. Hydrol., 399, 132-140, doi:10.1016/j.jhydrol.2010.12.041.

Labat, D., R. Ababou, and A. Mangin, 2000: Rainfall-runoff relationships for karstic springs. Part II: Continuous wavelet and discrete orthogonal multiresolution analyses. J. Hydrol., 238, 149-178, doi:10.1016/S0022-1694(00)00322-X.

Lin, J., and M. J. T. Smith, 2008: New perspectives and improvements on the symmetric extension filter bank for subband/ wavelet image compression. IEEE Trans. Image Process., 7, 177-180, doi:10.1109/TIP.2007.914223.

Lin, K., Y. He, X. Lei, and X. Chen, 2011: Climate change and its impact on runoff during 1956-2009 in Dongjiang basin (in Chinese). Ecol. Environ. Sci., 20 (12), 1783-1787.

Liong, S., and C. Sivapragasam, 2002: Flood stage forecasting with support vector machines. J. Amer. Water Resour. Assoc., 38, 173-186, doi:10.1111/j.1752-1688.2002.tb01544.x.

Liu, Y., and H. V. Gupta, 2007: Uncertainty in hydrologic modeling: Toward an integrated data assimilation framework. Water Resour. Res., 43, W07401, doi:10.1029/2006WR005756.

_, J. Brown, J. Demargne, and D.-J. Seo, 2011: Assessing timing errors in hydrologic predictions using cross wavelet analysis. J. Hydrol., 397, 210-224, doi:10.1016/j.jhydrol.2010.11.040.

— , and Coauthors, 2012: Advancing data assimilation in operational hydrologic forecasting: Progresses, challenges, and emerging opportunities. Hydrol. Earth Syst. Sci., 16, $3863-$ 3887, doi:10.5194/hess-16-3863-2012.

Liu, Z., P. Zhou, G. Chen, and L. Guo, 2014: Evaluating a coupled discrete wavelet transform and support vector regression for daily and monthly streamflow forecasting. J. Hydrol., 519, 2822-2831, doi:10.1016/j.jhydrol.2014.06.050.

Luo, L., and E. F. Wood, 2008: Use of Bayesian merging techniques in a multimodel seasonal hydrologic ensemble prediction system for the eastern United States. J. Hydrometeor., 9, 866884, doi:10.1175/2008JHM980.1.

Maheswaran, R., and R. Khosa, 2012a: Wavelet-Volterra coupled model for monthly stream flow forecasting. J. Hydrol., 450451, 320-335, doi:10.1016/j.jhydrol.2012.04.017.

$\longrightarrow$, and _ 2012b: Comparative study of different wavelets for hydrologic forecasting. Comput. Geosci., 46, 284-295, doi:10.1016/j.cageo.2011.12.015.

Mallat, S. G., 1989: A theory for multiresolution signal decomposition: The wavelet representation. IEEE Trans. Pattern Anal. Mach. Intell., 11, 674-693, doi:10.1109/34.192463.

McKerchar, A. I., and J. W. Delleur, 1974: Application of seasonal parametric linear stochastic models to monthly flow data. Water Resour. Res., 10, 246-255, doi:10.1029/WR010i002p00246.

Moradkhani, H., K. Hsu, H. V. Gupta, and S. Sorooshian, 2004: Improved streamflow forecasting using self-organizing radial basis function artificial neural networks. J. Hydrol., 295, 246262, doi:10.1016/j.jhydrol.2004.03.027.

Nalley, D., J. Adamowski, and B. Khalil, 2012: Using discrete wavelet transforms to analyze trends in streamflow and precipitation in Quebec and Ontario (1954-2008). J. Hydrol., 475, 204-228, doi:10.1016/j.jhydrol.2012.09.049.

Nash, J. E., and J. V. Sutcliffe, 1970: River flow forecasting through conceptual models. J. Hydrol., 10, 282-290, doi:10.1016/ 0022-1694(70)90255-6.

Neuman, S. P., and P. J. Wierenga, 2003: A comprehensive strategy of hydrologic modeling and uncertainty analysis for nuclear facilities and sites. NUREG/CR-6805, U.S. Nuclear Regulatory Commission Office of Nuclear Regulatory Research, $311 \mathrm{pp}$.

Niu, J., 2013: Precipitation in the Pearl River basin, south China: Scaling, regional patterns, and influence of large-scale climate anomalies. Stochastic Environ. Res. Risk Assess., 27, 12531268, doi:10.1007/s00477-012-0661-2.

NOAA, 2015: MEI index dataset. NOAA/ESRL/PSD, accessed 30 June 2015. [Available online at http://www.esrl.noaa.gov/ psd/enso/mei/table.html.]

Nourani, V., M. T. Alami, and M. H. Aminfar, 2009a: A combined neural-wavelet model for prediction of Ligvanchai watershed precipitation. Eng. Appl. Artif. Intell., 22, 466-472, doi:10.1016/ j.engappai.2008.09.003.

_ M. Komasi, and A. Mano, 2009b: A multivariate ANNwavelet approach for rainfall-runoff modeling. Water Resour. Manage., 23, 2877-2894, doi:10.1007/s11269-009-9414-5.

—, O. Kisi, and M. Komasi, 2011: Two hybrid artificial intelligence approaches for modeling rainfall-runoff process. J. Hydrol., 402, 41-59, doi:10.1016/j.jhydrol.2011.03.002.

—, A. Hosseini Baghanam, J. Adamowski, and M. Gebremicheal, 2013: Using self-organizing maps and wavelet transforms for space-time pre-processing of satellite precipitation and runoff data in neural network based rainfall-runoff modeling. J. Hydrol., 476, 228-243, doi:10.1016/ j.jhydrol.2012.10.054.

,,,--- and O. Kisi, 2014: Applications of hybrid waveletartificial intelligence models in hydrology: A review. J. Hydrol., 514, 358-377, doi:10.1016/j.jhydrol.2014.03.057.

Parrish, M. A., H. Moradkhani, and C. M. DeChant, 2012: Toward reduction of model uncertainty: Integration of Bayesian model averaging and data assimilation. Water Resour. Res., 48, W03519, doi:10.1029/2011WR011116.

Partal, T., and M. Küçük, 2006: Long-term trend analysis using discrete wavelet components of annual precipitations measurements in Marmara region (Turkey). Phys. Chem. Earth, 31, 1189-1200, doi:10.1016/j.pce.2006.04.043.

Pokhrel, P., Q. J. Wang, and D. E. Robertson, 2013: The value of model averaging and dynamical climate model predictions for improving statistical seasonal streamflow forecasts over Australia. Water Resour. Res., 49, 6671-6687, doi:10.1002/ wrcr.20449.

Quiroz, R., C. Yarlequé, A. Posadas, V. Mares, and W. W. Immerzeel, 2011: Improving daily rainfall estimation from NDVI using a wavelet transform. Environ. Modell. Software, 26, 201-209, doi:10.1016/j.envsoft.2010.07.006.

Raftery, A. E., T. Gneiting, F. Balabdaoui, and M. Polakowski, 2005: Using Bayesian model averaging to calibrate forecast ensembles. Mon. Wea. Rev., 133, 1155-1174, doi:10.1175/ MWR2906.1.

Rathinasamy, M., J. Adamowski, and R. Khosa, 2013: Multiscale streamflow forecasting using a new Bayesian model average based ensemble multi-wavelet Volterra non-linear method. J. Hydrol., 507, 186-200, doi:10.1016/j.jhydrol.2013.09.025.

Rojas, R., L. Feyen, and A. Dassargues, 2008: Conceptual model uncertainty in groundwater modeling: Combining generalized likelihood uncertainty estimation and Bayesian model averaging. Water Resour. Res., 44, W12418, doi:10.1029/2008WR006908.

Saji, N. H., and T. Yamagata, 2003: Possible impacts of Indian Ocean dipole mode events on global climate. Climate Res., 25, 151-169, doi:10.3354/cr025151.

_ B. N. Goswami, P. N. Vinayachandran, and T. Yamagata, 1999: A dipole mode in the tropical Indian Ocean. Nature, 401, 360-363. 
Sang, Y. F., Z. Wang, and C. Liu, 2013: Discrete wavelet-based trend identification in hydrologic time series. Hydrol. Processes, 27, 2021-2031, doi:10.1002/hyp.9356.

Sloughter, J. M., A. E. Raftery, T. Gneiting, and C. Fraley, 2007: Probabilistic quantitative precipitation forecasting using Bayesian model averaging. Mon. Wea. Rev., 135, 3209-3220, doi:10.1175/MWR3441.1.

Su, H., Q. Liu, and J. Li, 2011: Alleviating border effects in wavelet transforms for nonlinear time-varying signal analysis. $A d v$ Electr. Comput. Eng., 11, 55-60, doi:10.4316/aece.2011.03009.

Tiwari, M. K., and C. Chatterjee, 2010: Development of an accurate and reliable hourly flood forecasting model using wavelet-bootstrap-ANN (WBANN) hybrid approach J. Hydrol., 394, 458-470, doi:10.1016/j.jhydrol.2010.10.001.

Vapnik, V., 2000: The Nature of Statistical Learning Theory. 2nd ed. Springer, $314 \mathrm{pp}$

_, S. Golwich, and A. J. Smola, 1997: Support vector method for function approximation, regression estimation, and signal processing. Advances in Neural Information Processing Systems 9, M. Mozer, M. I. Jordan, and T. Petsche, Eds., MIT Press, 281-287.

Vogel, R. M., and N. M. Fennessey, 1995: Flow duration curves II: A review of applications in water resources planning. J. Amer. Water Res. Assoc., 31, 1029-1039, doi:10.1111/ j.1752-1688.1995.tb03419.x.

Vrugt, J., and B. Robinson, 2007: Treatment of uncertainty using ensemble methods: Comparison of sequential data assimilation and Bayesian model averaging. Water Resour. Res., 43, W01411, doi:10.1029/2005WR004838.

Wang, Q. J., and D. E. Robertson, 2011: Multisite probabilistic forecasting of seasonal flows for streams with zero value occurrences. Water Resour. Res., 47, W02546, doi:10.1029/2010WR009333.
Wang, W., and J. Ding, 2003: Wavelet network model and its application to the prediction of the hydrology. Nat. Sci., 1 (1), 67-71.

Wang, W.-C., K. W. Chau, C. T. Cheng, and L. Qiu, 2009: A comparison of performance of several artificial intelligence methods for forecasting monthly discharge time series. J. Hydrol., 374, 294-306, doi:10.1016/j.jhydrol.2009.06.019.

Wang, Z., X. Chen, and T. Yan, 2010: Runoff variation and its impacting factors in the Dongjiang River basin during 19562005 (in Chinese with English abstract). J. Nat. Resour., 25 (8), 1365-1374.

Wei, C., and J. Roan, 2012: Retrievals for the rainfall rate over land using Special Sensor Microwave Imager data during tropical cyclones: Comparisons of scattering index, regression, and support vector regression. J. Hydrometeor., 13, 1567-1578, doi:10.1175/JHM-D-11-0118.1.

Wöhling, T., and J. A. Vrugt, 2008: Combining multiobjective optimization and Bayesian model averaging to calibrate forecast ensembles of soil hydraulic models. Water Resour. Res., 44, W12432, doi:10.1029/2008WR007154.

Wolter, K., and M. S. Timlin, 2011: El Niño-Southern Oscillation behaviour since 1871 as diagnosed in an extended multivariate ENSO index (MEI.ext). Int. J. Climatol., 31, 1074-1087, doi:10.1002/joc.2336.

Wu, Y., and J. Chen, 2013: Investigating the effects of point source and nonpoint source pollution on the water quality of the East River (Dongjiang) in south China. Ecol. Indic., 32, 294-304, doi:10.1016/j.ecolind.2013.04.002.

Yu, P. S., S. T. Chen, and I. F. Chang, 2006: Support vector regression for real-time flood stage forecasting. J. Hydrol., 328, 704-716, doi:10.1016/j.jhydrol.2006.01.021. 